Pure and Applied Mathematics Quarterly

Volume 5, Number 3

(Special Issue: In honor of

Friedrich Hirzebruch, Part 2 of 2)

1161-1199, 2009

\title{
Relative Proportionality for Subvarieties of Moduli Spaces of K3 and Abelian Surfaces
}

\author{
S. Müller-Stach, E. Viehweg and K. Zuo \\ To Friedrich Hirzebruch
}

\begin{abstract}
The relative proportionality principle of Hirzebruch and Höfer was discovered in the case of compactified ball quotient surfaces $X$ when studying curves $C \subset X$. It can be expressed as an inequality which attains equality precisely when $C$ is an induced quotient of a subball. A similar inequality holds for curves on Hilbert modular surfaces. In this paper we prove a generalization of this result to subvarieties of Shimura varieties of orthogonal type, i.e. locally symmetric spaces of type $\mathcal{M}=\Gamma \backslash \mathrm{SO}(n, 2) / K$. Furthermore we study the "inverse problem" of deciding when an arbitrary subvariety $Z$ of $\mathcal{M}$ is of Hodge type, provided it contains sufficiently many divisors $W_{i}$ which are of Hodge type and satisfy relative proportionality.

Keywords: Hirzebruch-Höefer proportionality, Shimura varieties, Shimura curves, Hilbert modular varieties, ball quotients.

Let $\mathcal{M}$ denote a connected Shimura variety of Hodge type associated to a reductive Lie group $G \subset \mathrm{Sp}_{2 g}$ of Hermitian type defined over $\mathbb{Q}$. A subvariety of $\mathcal{M}$ is called special or a subvariety of Hodge type, if it is induced by an algebraic subgroup $G_{1} \hookrightarrow G$ of Hermitian type. In particular zero dimensional special subvarieties are just the CM-points.
\end{abstract}

Received January 20, 2008.

This work has been supported by the DFG-Leibniz program and by the SFB/TR 45 "Periods, moduli spaces and arithmetic of algebraic varieties". 
As it is well known, a subvariety of Hodge type contains a dense set of CMpoints. The André-Oort conjecture states the converse, hence that an irreducible variety $Z$ of $\mathcal{M}$ is a subvariety of Hodge type, if the CM points in $Z$ are Zariski dense. Recently Klingler and Yafaev [KY06] have given a proof of this conjecture, assuming the generalized Riemann Hypothesis. The André-Oort conjecture implies immediately that a subvariety $Z$ of $\mathcal{M}$ which contains a Zariski dense set of subvarieties of Hodge type must itself be special. We will restrict ourselves to the moduli space $\mathcal{M}$ of polarized K3 or abelian surfaces, more generally subvarieties of Shimura varieties of orthogonal type, i.e. locally symmetric spaces of type $\mathcal{M}=\Gamma \backslash \mathrm{SO}(n, 2) / K$ with $\Gamma$ a neat arithmetic group. For $Z \subset \mathcal{M}$ we will show that a "big finite subset" $\left\{W_{i}\right\}_{i \in I}$ of subvarieties of Hodge type of codimension one is sufficient to force $Z$ to be special. One way to formulate the bigness of the set of subvarieties would be to require the natural map

$$
\pi_{1}\left(\bigcup_{i \in I} W_{i}\right) \longrightarrow \pi_{1}(Z)
$$

to be surjective, for a suitable choice of base points. Instead we will consider certain compactifications $\bar{Z}$ of $Z$, and require $\# I$ to be large compared with its Picard number $\rho(\bar{Z})$ and with the number $\delta\left(S_{\bar{Z}}\right)$ of different two by two intersections $S_{i} \cap S_{j}$ of irreducible components $S_{i}$ and $S_{j}$ of $S_{\bar{Z}}$.

A second aspect is the understanding of the "relative proportionality", a numerical condition satisfied by subvarieties of Hodge type $W$ of $Z$, provided the universal covering $\tilde{Z}$ of $Z$ is a bounded symmetric domain. The proportionality principle has been established by Hirzebruch in [Hi58] for projective manifolds $Z$, and it has been generalized by Mumford in [Mu77] to the quasi-projective case. To this aim, Mumford used a particularly nice toroidal compactification $\bar{Z}$ of $Z$, constructed in [AMRT75] and extensions of the Hodge bundles to $\bar{Z}$.

In the mid 1980's Hirzebruch and Höfer have obtained the relative proportionality inequality for an algebraic curve $\bar{C}$ on an algebraic surface $\bar{Y}$ with universal covering $\tilde{Y}$ a complex ball (see [BHH87, page 259 and 265], for example). A similar inequality holds for curves on Hilbert modular surfaces, and for special curves the equality was already verified in $[\mathrm{HZ73}, \S 4]$.

Theorem 0.1 (Hirzebruch [HZ73]; Hirzebruch, Höfer [BHH87]). Assume that $S_{\bar{Y}}=\bar{Y} \backslash Y$ is a strict normal crossing divisor. Then for a non-singular curve $\bar{C} \subset \bar{Y}$ and for the reduced boundary divisor $S_{\bar{C}}=\left(\bar{C} \cap S_{\bar{Y}}\right)_{\text {red }}$ one has the relative 
proportionality inequality saying that

$$
2 \cdot \bar{C} \cdot \bar{C}+2 \cdot \operatorname{deg}\left(S_{\bar{C}}\right) \geq-K_{\bar{Y}} \cdot \bar{C}+S_{\bar{Y}} \cdot \bar{C},
$$

if $Y$ is a Hilbert modular surface, and

$$
3 \cdot \bar{C} \cdot \bar{C}+3 \cdot \operatorname{deg}\left(S_{\bar{C}}\right) \geq-K_{\bar{Y}} \cdot \bar{C}+2 \cdot S_{\bar{Y}} \cdot \bar{C},
$$

if $Y$ is a ball quotient.

If the compactification $\bar{Y}$ is a Mumford compactification, or more generally if $\Omega_{\bar{Y}}^{1}\left(\log S_{\bar{Y}}\right)$ is numerically effective (nef) and if $\omega_{\bar{Y}}\left(S_{\bar{Y}}\right)$ is ample with respect to $Y$, then the equality in (0.1) or in (0.2) implies that $\tilde{C}$ is a complex subball of $\tilde{Y}$.

In [BHH87] these inequalities are stated only in the case where $\bar{C} \cap S_{\bar{Y}}$ intersect transversally. Then they simplify to $2 \cdot \bar{C} \cdot \bar{C} \geq-\left(K_{\bar{Y}}+S_{\bar{Y}}\right) \cdot \bar{C}$ on Hilbert modular surfaces and $3 \cdot \bar{C} \cdot \bar{C} \geq-\left(K_{\bar{Y}}+S_{\bar{Y}}\right) \cdot \bar{C}$ on ball quotients.

In Section 2 we will prove and generalize those inequalities to certain higher dimensional Shimura varieties $\mathcal{M}$ which are uniformized by a variation of Hodge structures $\mathbb{V}$ of weight two. Assuming that the local monodromies at infinity are unipotent, we consider to this aim the Higgs bundle $(E, \theta)$ induced by the Deligne extension of $\mathbb{V}$ to $\overline{\mathcal{M}}$, as explained in the Notations 0.4 , and the corresponding Griffiths-Yukawa coupling. For the generalizations of Theorem 0.1, stated in Theorem 2.3 we will allow $\mathcal{M}$ to be a Shimura variety of complex ball type, i.e. $\tilde{\mathcal{M}}=\mathrm{SU}(n, 1) / \mathrm{U}(n)$, or of type $\mathrm{SO}(n, 2)$, i.e. $\tilde{\mathcal{M}}=\mathrm{SO}(n, 2) / \mathrm{O}(n) \times \mathrm{U}(1)$, and we replace the curve $C$ by a submanifold $Z$. We will distinguish the different cases corresponding to (0.1) and (0.2) in Theorem 0.1 by posing conditions on the Griffiths-Yukawa coupling.

For example, a curve $C$ on a Hilbert modular surface $Y$ has Griffiths-Yukawa coupling $\theta_{\bar{C}}^{(2)} \neq 0$, whereas for curves in a ball quotient $Y$ it will vanish, since already $\theta_{\bar{Y}}^{(2)}=0$. The remaining case, where the Griffiths-Yukawa coupling satisfies $\theta_{\bar{C}}^{(2)}=0$ but $\theta_{\bar{Y}}^{(2)} \neq 0$, only occurs on fake Hilbert modular surfaces, i.e. on products $X=C_{1} \times C_{2}$ of two curves of genus $g \geq 2$, and for $C$ a fiber of one of the projections. So to handle this case we should add in Theorem 2.3:

$$
\bar{C} \cdot \bar{C}+\operatorname{deg}\left(S_{\bar{C}}\right) \geq S_{\bar{Y}} \cdot \bar{C}, \quad \text { if } Y \text { is a product } C_{1} \times C_{2} .
$$

However the condition $\theta_{\bar{C}}^{(2)}=0$ on the product of two curves only occurs if $\bar{C}$ is the fiber of one of the projections, and hence $\bar{C} \cdot \bar{C}=0$ and $\operatorname{deg}\left(S_{\bar{C}}\right)=S_{\bar{Y}} \cdot \bar{C}$. 
Let us return to the problem of characterizing subvarieties of Hodge type in $\mathcal{M}$ by the existence of a big set of special subvarieties. Starting with Section 3 we will restrict ourselves to the case where $\mathcal{M}$ is of type $\mathrm{SO}(n, 2)$, and we will consider $W \subset Z \subset \mathcal{M}$, with $W$ a Shimura variety and $Z$ unknown. Doing so for Shimura curves $C$ on surfaces $Y \subset \mathcal{M}$, we get similar expressions as (0.1), (0.2) or (0.3) with the sign reversed:

Theorem 0.2. Assume that $\bar{Y}$ is a projective surface, $S_{\bar{Y}}$ a strict normal crossing divisor and $Y=\bar{Y} \backslash S_{\bar{Y}}$, with $\Omega_{\bar{Y}}^{1}\left(\log S_{\bar{Y}}\right)$ nef and $\omega_{\bar{Y}}\left(\log S_{\bar{Y}}\right)$ ample with respect to $Y$.

Consider a Shimura curve $C \subset Y$ and a closed embedding $Y \subset \mathcal{M}$. Assume (for simplicity) that the second embedding extends to $\bar{Y} \subset \overline{\mathcal{M}}$ for a Mumford compactification $\overline{\mathcal{M}}$ of $\mathcal{M}$. If $C$ is a Shimura curve, then one has:

$$
\begin{aligned}
& 2 \cdot \bar{C} \cdot \bar{C}+2 \cdot \operatorname{deg}\left(S_{\bar{C}}\right) \leq-K_{\bar{Y}} \cdot \bar{C}+S_{\bar{Y}} \cdot \bar{C} \quad \text { if } \theta_{\bar{C}}^{(2)} \neq 0 . \\
& 3 \cdot \bar{C} \cdot \bar{C}+3 \cdot \operatorname{deg}\left(S_{\bar{C}}\right) \leq-K_{\bar{Y}} \cdot \bar{C}+2 \cdot S_{\bar{Y}} \cdot \bar{C} \quad \text { if } \theta_{\bar{Y}}^{(2)}=0 \text {. } \\
& \bar{C} \cdot \bar{C}+\operatorname{deg}\left(S_{\bar{C}}\right) \leq S_{\bar{Y}} \cdot \bar{C} \quad \text { if } \theta_{\bar{C}}^{(2)}=0 \text { but } \theta_{\bar{Y}}^{(2)} \neq 0 .
\end{aligned}
$$

Again the first two inequalities generalize to the higher dimensional case (see Theorem 3.3). Now we can formulate a criterion for $Y$ to be a Shimura surface of Hodge type:

Theorem 0.3. Consider in Theorem 0.2 a finite set of curves $\left\{C_{i}\right\}_{i \in I}$, with

$$
\# I \geq\left(\rho(\bar{Y})+\delta\left(S_{\bar{Y}}\right)\right)^{2}+\rho(\bar{Y})+\delta\left(S_{\bar{Y}}\right)+1,
$$

where $\delta\left(S_{\bar{Y}}\right)$ is the number of double points on the boundary and where $\rho(\bar{Y})$ is the Picard number of $\bar{Y}$.

i) If for all $i \in I$

$$
2 \cdot \bar{C}_{i} \cdot \bar{C}_{i}+2 \cdot \operatorname{deg}\left(S_{\bar{C}_{i}}\right)=-K_{\bar{Y}} \cdot \bar{C}_{i}+S_{\bar{Y}} \cdot \bar{C}_{i}
$$

and $\theta_{\bar{C}_{i}}^{(2)} \neq 0$, then $Y$ is a Hilbert modular surface.

ii) If for all $i \in I$

$$
3 \cdot \bar{C}_{i} \cdot \bar{C}_{i}+3 \cdot \operatorname{deg}\left(S_{\bar{C}_{i}}\right)=-K_{\bar{Y}} \cdot \bar{C}_{i}+2 \cdot S_{\bar{Y}} \cdot \bar{C}_{i}
$$

and $\theta_{\bar{Y}}^{(2)}=0$, then $Y$ is a ball quotient. 
In both Theorems, 0.2 and 0.3 , one can allow the curves $C$ or $C_{i}$ to be deformations of Shimura curves in $\mathcal{M}$, as defined in 1.2. However such a deformation can only be non-trivial if $\theta_{\bar{C}}^{(2)}$ or $\theta_{\bar{C}_{i}}^{(2)}$ are zero.

As we will see in the proofs, a quasi-projective surface $Y \subset \mathcal{M}$ containing a Shimura curve $C$ of Hodge type (or its deformation) and satisfying relative proportionality in Theorem 0.3 i) or ii), looks in an infinitesimal neighborhood of $C$ like a Shimura surface of the corresponding type.

The corresponding statement in Theorem 4.4 will be formulated for submanifolds $Z \subset \mathcal{M}$ of arbitrary dimension, but the codimension of the Shimura subvarieties $W \subset Z$, replacing the curves $C$, still has to be one.

Notations 0.4. We consider a projective manifold $\bar{Z}$, a reduced strict normal crossing divisor $S_{\bar{Z}}$ and a variation of Hodge structures $\mathbb{V}$ on $Z=\bar{Z} \backslash S_{\bar{Z}}$ of weight $k$. Even if not stated we will always assume that the local monodromies around the components of $S_{\bar{Z}}$ are unipotent, and that $\mathbb{V}$ is polarized. Let $\mathcal{V}$ be the Deligne extension of $\mathbb{V} \otimes_{\mathbb{C}} \mathcal{O}_{Z}$ to $\bar{Z}$. The $\mathcal{F}$-filtration on $Z$ extends to a filtration of $\mathcal{V}$ by subbundles, and the Gauß-Manin connection extends to a connection $\nabla$ with logarithmic poles on $\mathcal{V}$. Griffiths Transversality implies that $\nabla$ induces an $\mathcal{O}_{\bar{Z}}$-linear map

$\theta: E:=\bigoplus_{p=0}^{k} E^{p, k-p}=\mathfrak{G r}_{\mathcal{F}}(\mathcal{V}) \longrightarrow E \otimes \Omega_{\bar{Z}}^{1}\left(\log S_{\bar{Z}}\right)=\left(\bigoplus_{p=0}^{k} E^{p, k-p}\right) \otimes \Omega_{\bar{Z}}^{1}\left(\log S_{\bar{Z}}\right)$, with $\theta\left(E^{p, k-p}\right) \subset E^{p-1, k-p+1} \otimes \Omega_{\bar{Z}}^{1}\left(\log S_{\bar{Z}}\right)$. We will call $(E, \theta)$ the Higgs bundle induced by the Deligne extension of $\mathbb{V}$, and $\theta$ the Higgs field. If we want to indicate the base space, we will write $\left(E_{\bar{Z}}, \theta_{\bar{Z}}\right)$ instead of $(E, \theta)$.

The Higgs field is the direct sum of maps,

$$
\theta_{p, k-p}: E^{p, k-p} \longrightarrow E^{p-1, k-p+1} \otimes \Omega_{\bar{Z}}^{1}\left(\log S_{\bar{Z}}\right)
$$

Their composite

$$
\theta_{\bar{Z}}^{(k)}:=\left(\theta_{1, k-1} \otimes \mathrm{id}_{\Omega_{\bar{Z}}^{1}\left(\log S_{\bar{Z}}\right)}^{\otimes k-1}\right) \circ \cdots \circ\left(\theta_{k-1,1} \otimes \operatorname{id}_{\Omega_{\bar{Z}}^{1}\left(\log S_{\bar{Z}}\right)}\right) \circ \theta_{k, 0},
$$

called the Griffiths-Yukawa coupling, has image in $E^{0, k} \otimes S^{k}\left(\Omega_{\bar{Z}}^{1}\left(\log S_{\bar{Z}}\right)\right)$.

Let $\overline{\mathcal{M}}$ be a second projective manifold and let $\mathcal{M}$ be the complement of a reduced strict normal crossing divisor $S_{\overline{\mathcal{M}}}$. We will consider a morphism $\varphi: Z \rightarrow$ 
$\mathcal{M}$, generically finite over its image. We will denote the induced rational map $\bar{Z} \rightarrow \overline{\mathcal{M}}$ again by $\varphi$.

The rational map $\varphi: \bar{Z} \rightarrow \bar{M}$ is given by a morphism $\varphi_{0}$ on the complement $\bar{Z}_{0}$ of a codimension two subscheme. For a locally free sheaf $\mathcal{F}$ on $\overline{\mathcal{M}}$ we will write $\varphi^{*} \mathcal{F}$ for the maximal extension of the pullback $\varphi_{0}^{*} \mathcal{F}$ from $\bar{Z}_{0}$ to $\bar{Z}$. Correspondingly, If $B$ is a reduced divisor, $\varphi^{*} B$ will be the closure of $\varphi_{0}^{*} B$.

The inclusion $\left.T_{\bar{Z}_{0}}\left(-\log S_{\bar{Z}_{0}}\right) \rightarrow \varphi^{*} T_{\overline{\mathcal{M}}}\left(-\log S_{\overline{\mathcal{M}}}\right)\right|_{\bar{Z}_{0}}$ extends to $\bar{Z}$ and we define the logarithmic normal sheaf $\check{N}_{\bar{Z} / \bar{M}}$ by the exact sequence

$$
0 \longrightarrow T_{\bar{Z}}\left(-\log S_{\bar{Z}}\right) \longrightarrow \varphi^{*} T_{\overline{\mathcal{M}}}\left(-\log S_{\overline{\mathcal{M}}}\right) \longrightarrow \check{N}_{\bar{Z} / \overline{\mathcal{M}}} \longrightarrow 0 .
$$

Even if $\bar{Z}$ is a submanifold of $\overline{\mathcal{M}}$ the logarithmic normal sheaf might differ from the usual normal sheaf $\mathcal{N}_{\bar{Z} / \overline{\mathcal{M}}}$ defined as the cokernel of $T_{\bar{Z}} \rightarrow \varphi^{*} T_{\overline{\mathcal{M}}}$. Comparing the Chern classes one obtains for $\bar{Z} \subset \overline{\mathcal{M}}$

$$
\mathrm{c}_{1}\left(\check{N}_{\bar{Z} / \overline{\mathcal{M}}}\right)=\mathrm{c}_{1}\left(\mathcal{N}_{\bar{Z} / \overline{\mathcal{M}}}\right)-S_{\left.\overline{\mathcal{M}}\right|_{\bar{Z}}}+S_{\bar{Z}}
$$

More generally, assume that $\varphi: Z \rightarrow \mathcal{M}$ is étale over its image of degree $\operatorname{deg}(\varphi)$ and that $\operatorname{dim}(\overline{\mathcal{M}})=n=\operatorname{dim}(\bar{Z})+1$. Writing $\varphi(\bar{Z})$ for the closure of the image of $\bar{Z}$ in $\overline{\mathcal{M}}$, one finds for all divisors $L$ on $\overline{\mathcal{M}}$

$$
\mathrm{c}_{1}\left(\check{N}_{\bar{Z} / \overline{\mathcal{M}}}\right) \cdot\left(\varphi^{*} L\right)^{n-2}=\operatorname{deg}(\varphi) \cdot \varphi(\bar{Z})^{2} \cdot L^{n-2}-\left(\left.S_{\overline{\mathcal{M}}}\right|_{\varphi(\bar{Z})}-\left(\left.S_{\overline{\mathcal{M}}}\right|_{\varphi(\bar{Z})}\right)_{\mathrm{red}}\right) \cdot L^{n-2} .
$$

In fact, both sides are compatible with blowing ups of $\bar{Z}$ with centers in $S_{\bar{Z}}$. So we may assume that $\varphi$ is a morphism. The formula (0.9) holds, if $\varphi$ is injective, and the general case follows from the projection formula. Usually $\mathcal{M}$ and $W$ will denote Shimura varieties, and $Z$ will map to in $\mathcal{M}$, or $W$ will map to $Z$.

\section{Acknowledgements.}

We would like to thank Sheng-Li Tan and De-Qi Zhang for discussions on Lemma 4.1 .

\section{Shimura varieties of type $\mathrm{SO}(m, 2)$ and $\mathrm{SU}(m, 1)$}

Let us first recall some well known basic facts on connected Shimura varieties and their connected Shimura subvarieties (see also [An01] or [Mil04]). We 
consider

$H$ a connected semisimple group defined over $\mathbb{Q}$ and of Hermitian type,

$$
\begin{gathered}
K \subset H(\mathbb{R}) \text { a maximal compact subgroup } \\
X^{+}=H(\mathbb{R}) / K \text { a bounded symmetric domain for } H .
\end{gathered}
$$

Writing $H^{+}(\mathbb{R})$ for the connected component of 1 in $H(\mathbb{R})$, one can consider $X^{+}$ as a conjugacy class of 1-parameter subgroups $\mathrm{U}(1) \rightarrow H^{+}(\mathbb{R})$.

Choose any $\mathbb{Z}$-structure $H_{\mathbb{Z}}$ on $H$ and let $\Gamma \subset H(\mathbb{Q})$ be an arithmetic subgroup, i.e. a subgroup $\Gamma$ which is commensurable to $H_{\mathbb{Z}}(\mathbb{Z})$. In addition we will always assume that $\Gamma$ is neat. By a theorem of Baily-Borel the analytic space $\mathcal{M}^{\text {an }}:=$ $\Gamma \backslash X^{+}$admits the so-called Baily-Borel compactification $\overline{\mathcal{M}}^{*}=\mathcal{M} \cup \Delta^{*}$ by adding the cusps $\Delta^{*}$ at infinity in $\mathcal{M}$. Since $\mathcal{M}^{*}$ is projective, $\mathcal{M}^{\text {an }}$ has the structure of an algebraic variety $\mathcal{M}$ over $\mathbb{C}$, inducing the analytic space structure on $\mathcal{M}^{\text {an }}$. Since $\Gamma$ is torsion-free $\mathcal{M}$ is smooth.

We will call $\mathcal{M}$ a connected Shimura variety, although one sometimes requires in addition that $\Gamma \subset H_{\mathbb{Z}}(\mathbb{Z})$ is a congruence subgroup, i.e. that $\Gamma$ contains the kernel of $H_{\mathbb{Z}}(\mathbb{Z}) \rightarrow H_{\mathbb{Z}}(\mathbb{Z} / N \mathbb{Z})$ for some $N$.

Now let $G$ be a connected reductive group over $\mathbb{Q}$, and such that

$$
H=G^{a d}=G / Z(G)
$$

is of Hermitian type. Then $X^{+}$is a $G^{+}(\mathbb{R})$-conjugacy class of 1-parameter subgroups.

If we have another group $G_{1}$ of Hermitian type, and a homomorphism $G_{1} \rightarrow G$ sending conjugacy classes $X_{1}^{+} \rightarrow X^{+}$, then the map $X_{1}^{+} \rightarrow X^{+}$is holomorphic and totally geodesic by Satake.

A Shimura subvariety of Hodge type (also called special subvariety) is a component of the image of some $X_{1}^{+}$in $\mathcal{M}$.

The following theorem gives a characterization of the quasi-projective subvarieties of $\mathcal{M}$ which are Shimura subvarieties of Hodge type or deformations of those. There is also the more general notion of Kuga fiber spaces (used in [MVZ07]) and of their bases. These are subvarieties of Hodge type if they contain a point corresponding to a $\mathrm{CM}$ abelian variety. 
Theorem 1.1 (Abdulali [Abd94], Moonen [Mo98], see also [MVZ07], Section 1).

Let $W \subset \mathcal{M}$ be a closed algebraic totally geodesic embedding. Then $\mathcal{M}$ contains a Shimura subvariety of Hodge type, isomorphic to $W \times W^{\prime}$ (up to a finite étale cover). In particular, if $W$ is rigid, hence if $W^{\prime}$ is a point, $W$ is a Shimura subvariety of Hodge type.

Notations 1.2. We will consider Shimura varieties up to étale coverings so we will allow to replace $\Gamma$ by a subgroup of finite index, whenever necessary. By abuse of notations we will call $W$ a Shimura subvariety of Hodge type if $\sigma(W)$ has this property and if $\sigma: W \rightarrow \sigma(W)$ is étale.

A subvariety $\iota: W \hookrightarrow \mathcal{M}$ will be called a deformation of a Shimura subvariety of Hodge type, if there exists a connected scheme $W^{\prime}$, points $w_{1}^{\prime}, w_{2}^{\prime} \in W^{\prime}$ and a morphism $\Psi: W \times W^{\prime} \rightarrow \mathcal{M}$, such that $\iota=\left.\Psi\right|_{W \times\left\{w_{1}^{\prime}\right\}}$ and such that $\left.\Psi\right|_{W \times\left\{w_{2}^{\prime}\right\}}$ is an embedding whose image is a Shimura subvariety of Hodge type.

Next, following [Kud03], we introduce Shimura varieties of orthogonal type determined by the following data:

$$
\begin{gathered}
V,(,) \text { an inner product space over } \mathbb{Q} \text { of signature }(n, 2), \\
G=\mathrm{SO}(n, 2)=\left\{g \in \mathrm{SL}_{n+2} \mid(g(x), g(y))=(x, y), \forall x, y \in V\right\} .
\end{gathered}
$$

In number theory one often prefers to work with the isogenous group $\operatorname{GSpin}(V)$. One defines the $n$-dimensional complex space

$$
D=\{w \in V(\mathbb{C}) \mid(w, w)=0,(w, \bar{w})<0\} / \mathbb{C}^{*} \subset \mathbb{P}(V(\mathbb{C})),
$$

which is the union $D=D^{+} \cup D^{-}$of two copies of the bounded symmetric domain $\mathrm{SO}(n, 2) / \mathrm{O}(n) \times \mathrm{U}(1)$ of type IV, interchanged by complex conjugation.

Fixing a $\mathbb{Z}$-structure $G_{\mathbb{Z}}(\mathbb{Z})$ on $G$ and again a neat arithmetic subgroup $\Gamma \subset$ $G(\mathbb{Q})$, the quotient $\mathcal{M}:=\Gamma \backslash D^{+}$is called a connected Shimura variety of orthogonal type.

The inner product space $V,($,$) together with the \mathbb{Z}$-structure descends to a polarized variation of Hodge structure $\mathbb{V}$ with a $\mathbb{Z}$-structure of weight two over $\mathcal{M}$. The one-dimensional vector spaces $V_{w}$ lying over $[w] \in D^{+}$define the Hodge bundle $E^{2,0}$ and their complex conjugates $\bar{V}_{w}$ define $E^{0,2}$. The orthogonal complements of the span $\left\langle V_{w}, \bar{V}_{w}\right\rangle$, for $w \in D^{+}$define $E^{1,1}$. It is also known 
that the Kodaira-Spencer (or dual Higgs field) $\theta: E^{2,0} \otimes T_{\mathcal{M}} \longrightarrow E^{1,1}$ is an isomorphism.

Assuming that the local monodromies around the cusps are unipotent, and that $\Gamma$ is neat, Mumford studied in [Mu77] smooth toroidal compactifications $\overline{\mathcal{M}}$ with $S_{\overline{\mathcal{M}}}=\overline{\mathcal{M}} \backslash \mathcal{M}$ a normal crossing divisor, constructed in [AMRT75]. The Higgs bundle $(E, \theta)$ extends to a unique logarithmic Higgs bundle on $\overline{\mathcal{M}}$, denoted again by $(E, \theta)$. In fact, as discussed in [MVZ07, Section 2], the bundle $(E, \theta)$ coincides with the one induced by the Deligne extension of $\mathbb{V}$ and the induced dual Higgs field

$$
E^{2,0} \otimes T_{\overline{\mathcal{M}}}\left(-\log S_{\overline{\mathcal{M}}}\right) \longrightarrow E^{1,1}
$$

is still an isomorphism.

To define Shimura subvarieties of $\mathcal{M}$, as in [Kud03], one starts with a set of $\mathbb{Q}$-linearly independent vectors $x=\left\{x_{1}, \ldots, x_{r}\right\} \subset V(\mathbb{Q})$ such that the intersection matrix $\left(\left(x_{i}, x_{j}\right)\right)_{i, j}$ is positive definite. We define $V_{x}$ to be the orthogonal complement of the span $\left\langle x_{1}, \ldots, x_{r}\right\rangle$, and $G_{x}$ to be the stabilizer of the span $\left.<x_{1}, \ldots, x_{r}\right\rangle$. The operation on $V_{x}$ defines an isomorphism $G_{x} \cong \mathrm{SO}(n-r, 2)$.

The embedding $\mathrm{SO}(n-r, 2) \hookrightarrow \mathrm{SO}(n, 2)$ of groups induces the totally geodesic holomorphic embedding of the corresponding bounded symmetric domains

$$
\mathrm{SO}(n-r, 2) / \mathrm{O}(n-r) \times \mathrm{U}(1) \longleftrightarrow \mathrm{SO}(n, 2) / \mathrm{O}(n) \times \mathrm{U}(1) .
$$

The image of $\mathrm{SO}(n-r, 2) / \mathrm{O}(n-r) \times \mathrm{U}(1)$ in $\mathcal{M}=\Gamma \backslash \mathrm{SO}(n, 2) / \mathrm{O}(n) \times \mathrm{U}(1)$ is a Shimura subvariety $W$ of orthogonal type (see [Kud03], Page 4, (2.6) and (2.8)).

The pullback of the variation of Hodge structures $\mathbb{V}$ to $W$ decomposes as $\mathbb{W} \oplus \mathbb{U}$, where $\mathbb{U}$ corresponds to a unitary variation of Hodge structures of bidegree $(1,1)$ with the $\mathbb{Z}$-structure arising from the $\mathbb{Q}$-subspace $\left\langle x_{1}, \ldots, x_{r}\right\rangle$. Hence, after taking a finite étale base change we may assume $\mathbb{U}$ is trivial. Correspondingly, one obtains a decomposition of the Higgs bundle of the variation of Hodge structures

$$
\left(E_{W}^{2,0} \oplus E_{W}^{1,1} \oplus E_{W}^{0,2}, \theta\right) \oplus\left(\mathcal{O}_{W}^{\oplus r}, 0\right),
$$

where $E_{W}^{2,0}$ and $E_{W}^{0,2}$ are the restrictions of the invertible sheaves $E^{2,0}$ and $E^{0,2}$ to $W$. Choosing as above a smooth Mumford compactification $\bar{W}$ of $W$ with $S_{\bar{W}}=\bar{W} \backslash W$ a strict normal crossing divisor, the logarithmic Higgs field defines an isomorphism

$$
\theta: E_{\bar{W}}^{2,0} \otimes T_{\bar{W}}\left(-\log S_{\bar{W}}\right) \longrightarrow E_{\bar{W}}^{1,1} .
$$


The Shimura subvariety $W$ is rigid in $\mathcal{M}$, since the Griffiths-Yukawa coupling does not vanish [MVZ07]. Hence by Theorem 1.1 it is of Hodge type and of type $\mathrm{SO}(n-r, 2)$, or as we will sometimes say, of Hodge type for $\mathrm{SO}(n-r, 2)$.

A Shimura variety is of Hodge type for $\mathrm{SU}(n, 1)$, if the associated Hermitian symmetric space is the $n$-dimensional complex ball

$$
X^{+}=\mathrm{SU}(n, 1) / \mathrm{U}(n) .
$$

Remark 1.3. In this case the natural uniformizing variation of Hodge structures is of weight one and the Higgs bundle has the form $\left(H^{1,0} \oplus H^{0,1}, \tau\right)$, where $H^{1,0}$ is a line bundle and where $\tau: T_{\mathcal{M}} \otimes H^{1,0} \rightarrow H^{0,1}$ is an isomorphism.

However if $\Gamma \backslash \mathrm{SU}(n, 1) / \mathrm{U}(n)$ occurs as a Shimura subvariety of Hodge type in some Shimura variety $\mathcal{M}$ of type $\operatorname{SO}(n, 2)$, then the restriction $(E, \theta)$ of the uniformizing variation of Hodge structures on $\mathcal{M}$ will be of weight two. The corresponding Higgs bundles are related by

$$
E^{2,0}=H^{1,0}, \quad E^{1,1}=H^{0,1} \oplus H^{0,1 \vee} \text { and } E^{0,2}=H^{1,0 \vee} .
$$

Example 1.4. Kondo [K05] has constructed a moduli embedding of a compact Shimura surface of type SU $(2,1)$ (appearing in Deligne-Mostow's list as a component in the moduli space parameterizing Jacobian of genus 6 admitting CM of $\left.\mathbb{Q}\left(e^{\frac{2 \pi i}{5}}\right)\right)$ into a Shimura variety of type $\mathrm{SO}(10,2)$ parameterizing a subfamily of K3 surfaces.

Lemma and Notations 1.5. Let $\sigma: W \rightarrow \mathcal{M}=\Gamma \backslash \mathrm{SO}(n, 2) / \mathrm{O}(n) \times \mathrm{U}(1)$ be a generically finite morphism from a non-singular $m$-fold $W$ and let $\bar{W}$ be a projective compactification of $W$ with $S_{\bar{W}}=\bar{W} \backslash W$ a strict normal crossing divisor and with $\omega_{\bar{W}}\left(S_{\bar{W}}\right)$ nef and big. Assume that the local monodromies around the components of $S_{\bar{W}}$ are unipotent, and write $\sigma^{*} \mathbb{V}=\mathbb{W} \oplus \mathbb{U}$ where $\mathbb{U}$ is the largest unitary subvariation of Hodge structures of type $(1,1)$.

For the logarithmic Higgs bundle $\left(E_{\bar{W}}, \theta_{\bar{W}}\right)$ induced by the Deligne extension of $\mathbb{W}$ to $\bar{W}$, let $E_{\diamond}^{1,1}$ denote the image of the Higgs map

$$
\theta: T_{\bar{W}}\left(-\log S_{\bar{W}}\right) \otimes E_{\bar{W}}^{2,0} \longrightarrow E_{\bar{W}}^{1,1} .
$$

i) The following conditions are equivalent:

a) $E_{\diamond}^{1,1}=E_{\bar{W}}^{1,1}$.

a') For the Higgs bundle $\left(E_{W}, \theta_{W}\right)=\left.\left(E_{\bar{W}}, \theta_{\bar{W}}\right)\right|_{W}$ one has $\left.E_{\diamond}^{1,1}\right|_{W}=$ $E_{W}^{1,1}$. 
b) $W$ is a Shimura subvariety of Hodge type for $\mathrm{SO}(m, 2)$.

c) $W$ is a Shimura subvariety of Hodge type and the Griffiths-Yukawa coupling $\theta_{\bar{W}}^{(2)}$ of $\left(E_{\bar{W}}, \theta_{\bar{W}}\right)$ is non-zero.

Moreover, if the conditions a), b) and c) hold true,

$$
\mathrm{c}_{1}\left(\omega_{\bar{W}}\left(S_{\bar{W}}\right)\right)=m \cdot \mathrm{c}_{1}\left(E_{\bar{W}}^{2,0}\right) .
$$

ii) The following conditions are equivalent:

a) The Higgs bundle $\left(E_{\bar{W}}, \theta_{\bar{W}}\right)$ decomposes as a direct sum

$$
\left(E_{\bar{W}}, \theta_{\bar{W}}\right)=\left(E_{\bar{W}}^{2,0} \oplus E_{\diamond}^{1,1}, \theta_{\diamond}\right) \oplus\left(E_{\diamond}^{\prime 1,1} \oplus E_{\bar{W}}^{0,2}, \theta_{\diamond}^{\prime}\right),
$$

where $E_{\bar{W}}^{1,1}=E_{\diamond}^{1,1} \oplus E_{\diamond}^{\prime 1,1}$ and $E_{\diamond}^{\prime 1,1} \neq 0$.

a') The Higgs bundle $\left(E_{W}, \theta_{W}\right)=\left.\left(E_{\bar{W}}, \theta_{\bar{W}}\right)\right|_{W}$ decomposes as a direct sum

$$
\left(E_{W}, \theta_{W}\right)=\left(\left.E_{W}^{2,0} \oplus E_{\diamond}^{1,1}\right|_{W}, \theta_{\diamond}\right) \oplus\left(\left.E_{\diamond}^{\prime 1,1}\right|_{W} \oplus E_{W}^{0,2}, \theta_{\diamond}^{\prime}\right) .
$$

b) $W$ is a deformation of a Shimura subvariety of Hodge type for the group $\mathrm{SU}(m, 1)$.

c) $W$ is a deformation of a Shimura subvariety of Hodge type and the Griffiths-Yukawa coupling $\theta_{\bar{W}}^{(2)}$ of $\left(E_{\bar{W}}, \theta_{\bar{W}}\right)$ is zero.

Moreover, if the conditions a), b) and c) hold true,

$$
\mathrm{c}_{1}\left(\omega_{\bar{W}}\left(S_{\bar{W}}\right)\right)=(m+1) \cdot \mathrm{c}_{1}\left(E_{\bar{W}}^{2,0}\right),
$$

and if $\operatorname{dim}(W)>1$ then $W$ is rigid, hence of Hodge type.

Proof. Assume first, that $\sigma(W) \subset \mathcal{M}$ is a deformation of a Shimura subvariety of Hodge type. Then one has the isomorphism in i), a') or the decomposition in ii), a'). We will show, that this extends to $\bar{W}$, in particular this will imply that in i) or ii) the conditions a) and a') are equivalent.

Let $\bar{W}^{\prime}$ be a Mumford compactification and $S_{\bar{W}^{\prime}}=\bar{W}^{\prime} \backslash W^{\prime}$. Then as discussed in [MVZ07, Section 2] the image of the Higgs field $E_{\diamond, \bar{W}^{\prime}}^{1,1}$ is a direct factor of $E_{\bar{W}^{\prime}}^{1,1}$. Choose a third compactification $\hat{W}$ of $W$ which allows morphisms $\phi: \hat{W} \rightarrow \bar{W}$ and $\phi^{\prime}: \hat{W} \rightarrow \bar{W}^{\prime}$. Since the Deligne extension is compatible with pullbacks, one has

$$
\phi^{*} T_{\bar{W}}\left(-\log S_{\bar{W}}\right) \hookrightarrow \phi^{*} E_{\bar{W}}^{1,1} \otimes E_{\bar{W}}^{2,0^{-1}}=\phi^{\prime *} E_{\bar{W}^{\prime}}^{1,1} \otimes E_{\bar{W}^{\prime}}^{2,0^{-1}} \hookleftarrow \phi^{\prime *} T_{\bar{W}^{\prime}}\left(-\log S_{\bar{W}^{\prime}}\right) .
$$


The inclusion on the right hand side splits, hence we obtain an inclusion

$$
\phi^{*} T_{\bar{W}}\left(-\log S_{\bar{W}}\right) \hookrightarrow \phi^{*} T_{\bar{W}^{\prime}}\left(-\log S_{\bar{W}^{\prime}}\right) .
$$

This must be an isomorphism, since as in the proof of [MVZ07, Lemma 2.7] it is easy to see that

$$
\phi^{*} \omega_{\bar{W}}\left(S_{\bar{W}}\right)=\phi^{\prime *} \omega_{\bar{W}^{\prime}}\left(S_{\bar{W}^{\prime}}\right)
$$

So $\phi^{*} T_{\bar{W}}\left(-\log S_{\bar{W}}\right)$ is a direct factor of $\phi^{*} E_{\bar{W}}^{1,1} \otimes E_{\bar{W}}^{2,0-1}$, and hence

$$
T_{\bar{W}}\left(-\log S_{\bar{W}}\right) \hookrightarrow E_{\bar{W}}^{1,1} \otimes E_{\bar{W}}^{2,0^{-1}}
$$

splits, as claimed in i), a) and ii), a).

Assuming the condition a) in i) or ii), we will write

$$
\left(E_{\bar{W}}, \theta_{\bar{W}}\right)=\left(E_{\diamond}, \theta_{\diamond}\right) \oplus\left(E_{\diamond}^{\prime}, \theta_{\diamond}^{\prime}\right),
$$

where the first direct factor contains $E^{2,0}$, hence $T_{\bar{W}}\left(\log S_{\bar{W}}\right) \otimes E^{2,0}$ as well.

We will show next, that the existence of this splitting of Higgs bundles forces $W$ to be the deformation of a Shimura subvariety of Hodge type. The decompositions, corresponding to $\sigma^{*} \mathbb{V}=\mathbb{W} \oplus \mathbb{U}$ or to the one in part ii), are both orthogonal with respect to the Hodge metric on the universal covering $\mathcal{M}$. The restriction of the Higgs map

$$
\theta: T_{\bar{W}}\left(-\log S_{\bar{W}}\right) \longrightarrow E_{\diamond}^{1,1} \otimes E_{\bar{W}}^{2,0 \vee}
$$

to $W$ is then an isomorphism, and it can be identified with the differential

$$
d \sigma: T_{W} \longrightarrow d \sigma\left(T_{W}\right) \subset \sigma^{*} T_{\mathcal{M}} \simeq E_{\bar{W}}^{1,1} \otimes E_{\bar{W}}^{2,0 \vee} \oplus U^{1,1} \otimes E_{\bar{W}}^{2,0 \vee},
$$

where $U^{1,1}$ is the Higgs bundle associated to $\mathbb{U}$. Hence the image of $d \sigma$ is a holomorphic direct factor, and orthogonal with respect to the Hodge metric. Therefore $\sigma$ is étale over its image and the latter is a non-singular subvariety of $\mathcal{M}$. Since $\sigma(W) \subset \mathcal{M}$ is a complete submanifold with respect to the Hodge metric $h$ (i.e. every Cauchy sequence in the sub metric space $\left(W, h_{W}\right) \subset(\mathcal{M}, h)$ converges to a point in $W$ ), then [MVZ07, Claim 6.9] together with [He62, Theorem I.14.5] show that $\sigma(W) \hookrightarrow \mathcal{M}$ is a holomorphic totally geodesic embedding. Then $W$ is either uniformized by $\mathrm{SO}(m, 2) / \mathrm{O}(m) \times \mathrm{U}(1)$ or by $\mathrm{SU}(m, 1) / \mathrm{U}(m)$, where $\mathrm{SO}(m, 2)$ respectively $\mathrm{SU}(m, 1)$ is the non-compact factor of the Zariski closure of the monodromy group. By Remark 1.3 the Griffiths-Yukawa coupling is zero for $W$ of type $\mathrm{SU}(m, 1)$ and non-zero for $W$ of type $\mathrm{SO}(m, 2)$. 
The non-vanishing of the Griffiths-Yukawa coupling implies the rigidity of $W$, and by Theorem $1.1 \mathrm{~W}$ is a Shimura variety of Hodge type in this case.

By [SZ91] the same holds true if the Griffiths-Yukawa coupling vanishes, and if $\operatorname{dim}(W)>1$.

Returning to the splitting in (1.1) one has $E_{\diamond}^{0,2}=E^{0,2}$ if and only if the Griffiths-Yukawa coupling does not vanish. By the choice of $\mathbb{W}$ this is equivalent to $E_{\diamond}^{\prime}=0$. So we verified the equivalence of the conditions a), b) and c) in i) and in ii).

It remains to verify the description of $\mathrm{c}_{1}\left(\omega_{\bar{W}}\left(S_{\bar{W}}\right)\right)$. In i) we have seen already that $E_{\diamond}^{0,2}=E^{0,2}$, hence $E_{\diamond}^{\prime}$ is concentrated in bidegree $(1,1)$ and $\theta_{\diamond}^{\prime}=0$. By the choice of $\mathbb{W}$ this is only possible if $E_{\diamond}^{\prime}=0$. Then

$$
\begin{gathered}
\mathrm{c}_{1}\left(E_{\bar{W}}^{2,0} \oplus E_{\diamond}^{1,1} \oplus E_{\bar{W}}^{0,2}\right)=\mathrm{c}_{1}\left(E_{\diamond}^{1,1}\right)=0 \\
\text { and } \quad \mathrm{c}_{1}\left(T_{\bar{W}}\left(-\log S_{\bar{W}}\right)\right)+m \cdot \mathrm{c}_{1}\left(E_{\bar{W}}^{2,0}\right)=0 .
\end{gathered}
$$

In Case ii) the Griffiths-Yukawa coupling is zero. So the Higgs subbundle $\left(E_{\diamond}, \theta_{\diamond}\right)$ is concentrated in bidegrees $(2,0)$ and $(1,1)$. Since $\left(E_{\bar{W}}, \theta_{\bar{W}}\right)$ is self dual, one finds that $E_{\diamond}^{\prime 1,1}=E_{\diamond}^{1,1 \vee}$. For the Chern classes this implies that

$$
\begin{gathered}
\mathrm{c}_{1}\left(E_{\bar{W}}^{2,0} \oplus E_{\diamond}^{1,1}\right)=0 \quad \text { and hence } \\
\mathrm{c}_{1}\left(T_{\bar{W}}\left(-\log S_{\bar{W}}\right)\right)+(m+1) \cdot \mathrm{c}_{1}\left(E_{\bar{W}}^{2,0}\right)=0 .
\end{gathered}
$$

\section{Remarks 1.6.}

1. The Shimura subvarieties in Lemma 1.5 include all rigid Shimura subvarieties of Shimura varieties of orthogonal type.

2. For $n=19 \mathcal{M}$ is the moduli scheme of polarized K3 surfaces [KS67]. The Kummer construction identifies $\mathcal{A}_{2}$ with a Shimura subvariety of $\mathrm{SO}(3,2)$ type. For $n=1$ and 2 one recovers modular curves, Hilbert modular surfaces and their quaternionic versions [Kud03].

3. If a Satake embedding $\mathcal{M} \rightarrow \mathcal{A}_{g}$ into a Shimura variety of $\operatorname{Sp}(2 g, \mathbb{R})$-type (i.e. into the moduli space of polarized abelian varieties with a suitable level structure) is of Hodge type, then it maps Shimura subvarieties of 
Hodge type to Shimura subvarieties of Hodge type [Abd94]. The KugaSatake construction, see [KS67] and [vG00], provides us with such an embedding.

\section{Hirzebruch-Höfer's Relative proportionality on Shimura VARIETIES OF TYPE $\mathrm{SU}(n, 1)$ OR $\mathrm{SO}(n, 2)$.}

In this section we will study subvarieties $Z$ of a Shimura variety $\mathcal{M}$ of type $\mathrm{SO}(n, 2)$ or $\mathrm{SU}(n, 1)$. We want to understand numerical conditions on natural sheaves on certain compactifications, generalizing the relative Hirzebruch-Höfer Proportionality stated in Theorem 0.1 .

Assumptions and Notations 2.1. Let $\mathcal{M}$ be a Shimura variety of type $\operatorname{SO}(n, 2)$ or $\mathrm{SU}(n, 1)$, and let $\overline{\mathcal{M}}$ be a smooth Mumford compactification of $\mathcal{M}$ with $S_{\overline{\mathcal{M}}}=\overline{\mathcal{M}} \backslash \mathcal{M}$ a strict normal crossing divisor. We denote by $\mathbb{V}$ the uniformizing weight two variation of Hodge structures on $\mathcal{M}$, and we will assume that the local monodromies around the components of $S_{\overline{\mathcal{M}}}$ and $S_{\bar{Z}}$ are unipotent. We write again $\mathbb{V}=\mathbb{W} \oplus \mathbb{U}$ where $\mathbb{U}$ is the maximal unitary subvariation of Hodge structures.

As in the Notations 0.4 let $\bar{Z}$ be a smooth projective $d$-dimensional variety, $S_{\bar{Z}}$ a reduced strict normal crossing divisor on $\bar{Z}$ and write $Z=\bar{Z} \backslash S_{\bar{Z}}$. We consider a morphism $\varphi: Z \rightarrow \mathcal{M}$ generically finite over its image and the induced rational map $\bar{Z} \rightarrow \overline{\mathcal{M}}$ again denoted by $\varphi$.

The Higgs bundle of the weight two variation of Hodge structures $\mathbb{W}$ on $\mathcal{M}$ will be denoted by $\left(E_{\overline{\mathcal{M}}}, \theta_{\overline{\mathcal{M}}}\right)$, whereas the Higgs bundle of the pullback of $\mathbb{W}$ to $Z$ is written as $\left(E_{Z}, \theta_{Z}\right)$. Let $\left(E_{\bar{Z}}, \theta_{\bar{Z}}\right)$ be the Higgs bundle induced by the Deligne extension of $\varphi^{*} \mathbb{W}$, so $\left(E_{Z}, \theta_{Z}\right)=\left.\left(E_{\bar{Z}}, \theta_{\bar{Z}}\right)\right|_{Z}$.

We will assume that $\Omega_{\bar{Z}}^{1}\left(\log S_{\bar{Z}}\right)$ nef and that $\omega_{\bar{Z}}\left(S_{\bar{Z}}\right)$ is ample with respect to $Z$.

The assumption, that $\Omega_{\bar{Z}}^{1}\left(\log S_{\bar{Z}}\right)$ is nef and that $\omega_{\bar{Z}}\left(S_{\bar{Z}}\right)$ is ample with respect to $Z$, will allow to apply Yau's Uniformization Theorem ([Ya93], see also [VZ05, Section 1]) to $Z$. As discussed in [VZ05, Lemma 4.1] and [MVZ07, §2] this assumption automatically holds true for compact submanifolds of Shimura varieties, and it holds if $Z$ is a Shimura subvariety of Hodge type, and $\bar{Z}$ a Mumford compactification. 
In general, for a rational map between manifolds, the pullback of the logarithmic tangent sheaf will not be locally free. However, since in our situation $\overline{\mathcal{M}}$ is a Mumford compactification of a Shimura variety this will be the case.

Lemma 2.2. We keep the assumptions made in 2.1.

a. The sheaf $\varphi^{*} T_{\overline{\mathcal{M}}}\left(-\log S_{\overline{\mathcal{M}}}\right)$ is locally free and isomorphic to a direct factor $E_{\diamond \bar{Z}}^{1,1} \otimes E_{\bar{Z}}^{2,0^{-1}}$ of $E_{\bar{Z}}^{1,1} \otimes E_{\bar{Z}}^{2,0^{-1}}=E_{\bar{Z}}^{1,1} \otimes E_{\bar{Z}}^{0,2}$.

b. If $\mathcal{M}$ is of type $\mathrm{SO}(n, 2)$, then $E_{\bar{Z}}^{1,1}=E_{\diamond \bar{Z}}^{1,1}$.

c. If $\mathcal{M}$ is of type $\mathrm{SU}(n, 1)$, then $E_{\bar{Z}}^{1,1}=E_{\diamond \bar{Z}}^{1,1} \oplus E_{\diamond \bar{Z}}^{1,1^{\vee}}$.

d. If $Z$ is the deformation of a Shimura subvariety of Hodge type, then $\check{N}_{\bar{Z} / \overline{\mathcal{M}}}$ is locally free and

$$
\varphi^{*} T_{\overline{\mathcal{M}}}\left(-\log S_{\overline{\mathcal{M}}}\right) \cong T_{\bar{Z}}\left(-\log S_{\bar{Z}}\right) \oplus \check{N}_{\bar{Z} / \overline{\mathcal{M}}}
$$

Proof. If $\varphi$ is an isomorphism, hence if $\bar{Z}=\overline{\mathcal{M}}$, the properties a), b) and c) have been verified in Lemma 1.5.

Let $\bar{Z}_{0}$ denote the largest open subscheme of $\bar{Z}$ for which $\left.\varphi^{-1}\left(S_{\overline{\mathcal{M}}}\right)\right|_{\bar{Z}_{0}}$ is a non-singular divisor and $\left.\varphi\right|_{\bar{Z}_{0}}$ a morphism. The Deligne extension is compatible with pullback under morphisms, and a), b) and c) hold true on $\bar{Z}_{0}$. Knowing this, and using the fact that the Higgs bundles induced by the Deligne extension of a variation of Hodge structures are locally free, one obtains a) and the description of the Higgs bundles in b) and c) extend to $\bar{Z}$.

The decomposition in Part d) follows, since in this case both,

$$
T_{\bar{Z}}\left(-\log S_{\bar{Z}}\right) \quad \text { and } \quad \varphi^{*} T_{\overline{\mathcal{M}}}\left(-\log S_{\overline{\mathcal{M}}}\right),
$$

are direct factors of $E_{\bar{Z}}^{1,1} \otimes E_{\bar{Z}}^{2,0^{-1}}$.

We will need the Simpson correspondence, hence the notion of slopes of coherent sheaves. Let $\mathcal{L}$ be an invertible sheaf, nef and ample with respect to $Z$. For any rank $r$ coherent sheaf $\mathcal{F}$ on $\bar{Z}$ define the degree and the slope with respect to $\mathcal{L}$ as

$$
\operatorname{deg}_{\mathcal{L}}(\mathcal{F}):=c_{1}(\mathcal{F}) \cdot c_{1}(\mathcal{L})^{d-1} \quad \text { and } \quad \mu_{\mathcal{L}}:=\frac{\operatorname{deg}_{\mathcal{L}}(\mathcal{F})}{r} .
$$

As we will see in the next Theorem, the generalized Hirzebruch-Höfer inequality is an inequality of Arakelov type similar to those considered in [STZ03] and [VZ03] 
over curves and in [VZ05] and [MVZ07] for variations of Hodge structures of weight one.

Theorem 2.3. (Hirzebruch-Höfer's relative proportionality inequality) Keeping the assumptions and notations stated in 2.1, one finds:

i) If $\mathcal{M}$ is of $\mathrm{SO}(n, 2)$-type and if the Griffiths-Yukawa coupling $\theta_{\bar{Z}}^{2} \neq 0$ then

$$
\begin{aligned}
& d \cdot \operatorname{deg}_{\omega_{\bar{Z}}\left(S_{\bar{Z}}\right)}\left(\check{N}_{\bar{Z} / \bar{M}}\right)+(n-d) \cdot \operatorname{deg}_{\omega_{\bar{Z}}\left(S_{\bar{Z}}\right)}\left(\Omega_{\bar{Z}}^{1}\left(\log S_{\bar{Z}}\right)\right)= \\
& n \cdot\left(\operatorname{deg}_{\omega_{\bar{Z}}\left(S_{\bar{Z}}\right)}\left(\Omega_{\bar{Z}}^{1}\left(\log S_{\bar{Z}}\right)\right)-d \cdot \operatorname{deg}_{\omega_{\bar{Z}}\left(S_{\bar{Z}}\right)}\left(E_{\bar{Z}}^{2,0}\right)\right) \geq 0 .
\end{aligned}
$$

The equality implies that $Z$ is a Shimura subvariety of $\mathcal{M}$ of Hodge type for $\mathrm{SO}(d, 2)$.

ii) If $\mathcal{M}$ is of type $\mathrm{SO}(n, 2)$ and if the Griffiths-Yukawa coupling $\theta_{\bar{Z}}^{2}$ is zero then

$$
\begin{aligned}
(d+1) \cdot \operatorname{deg}_{\omega_{\bar{Z}}\left(S_{\bar{Z}}\right)}\left(\check{N}_{\bar{Z} / \overline{\mathcal{M}}}\right)+(n-d-1) \cdot \operatorname{deg}_{\omega_{\bar{Z}}\left(S_{\bar{Z}}\right)}\left(\Omega_{\bar{Z}}^{1}\left(\log S_{\bar{Z}}\right)\right)= \\
n \cdot\left(\operatorname{deg}_{\omega_{\bar{Z}}\left(S_{\bar{Z}}\right)}\left(\Omega_{\bar{Z}}^{1}\left(\log S_{\bar{Z}}\right)\right)-(d+1) \cdot \operatorname{deg}_{\omega_{\bar{Z}}\left(S_{\bar{Z}}\right)}\left(E_{\bar{Z}}^{2,0}\right)\right) \geq 0 .
\end{aligned}
$$

The equality implies that $Z$ is either the the deformation of a Shimura curve in $\mathcal{M}$ or, if $\operatorname{dim}(Z)>1$, that $Z$ is a Shimura subvariety of $\mathcal{M}$ of Hodge type for $\mathrm{SU}(d, 1)$.

As in Remark 1.3 on a Shimura variety $\mathcal{M}$ of type $\mathrm{SU}(n, 1)$ we consider the weight two variation of Hodge structures with logarithmic Higgs bundle $\left(E_{\overline{\mathcal{M}}}, \theta_{\overline{\mathcal{M}}}\right)$ given as the direct sum of $(H, \tau)$ and its dual.

\section{Addendum 2.4.}

iii) If $\mathcal{M}$ is of type $\mathrm{SU}(n, 1)$, then the Griffiths-Yukawa coupling $\theta_{\bar{Z}}^{2}$ is zero and

$$
\begin{aligned}
& (d+1) \cdot \operatorname{deg}_{\omega_{\bar{Z}}\left(S_{\bar{Z}}\right)}\left(\check{N}_{\bar{Z} / \bar{M}}\right)+(n-d) \cdot \operatorname{deg}_{\omega_{\bar{Z}}\left(S_{\bar{Z}}\right)}\left(\Omega_{\bar{Z}}^{1}\left(\log S_{\bar{Z}}\right)\right)= \\
& (n+1) \cdot\left(\operatorname{deg}_{\omega_{Z}(S)}\left(\Omega_{\bar{Z}}^{1}\left(\log S_{\bar{Z}}\right)\right)-(d+1) \cdot \operatorname{deg}_{\omega_{\bar{Z}}\left(S_{\bar{Z}}\right)}\left(E_{\bar{Z}}^{2,0}\right)\right) \geq 0 .
\end{aligned}
$$

Again the equality implies that $Z$ is either the deformation of a Shimura curve in $\mathcal{M}$ or, if $\operatorname{dim}(Z)>1$, that $Z$ is a Shimura subvariety of $\mathcal{M}$ of Hodge type for $\mathrm{SU}(d, 1)$. 
Proof of Theorem 2.3 and Addendum 2.4. All the arguments will concern $\bar{Z}$, so for simplicity we will drop the lower index $\bar{Z}$ for the Higgs bundles on $\bar{Z}$ and we will write deg and $\mu$ instead of $\operatorname{deg}_{\omega_{\bar{Z}}\left(S_{\bar{Z}}\right)}$ and $\mu_{\omega_{\bar{Z}}\left(S_{\bar{Z}}\right)}$.

Let us first show the equalities on the left hand sides. We know that

$$
\varphi^{*} T_{\overline{\mathcal{M}}}\left(-\log S_{\overline{\mathcal{M}}}\right)=E_{\diamond}^{1,1} \otimes E^{0,2}
$$

is a direct factor of $E^{1,1} \otimes E^{0,2}$. In Theorem 2.3 both coincide and $\operatorname{deg}\left(E^{1,1}\right)=0$. The exact sequence (0.7) together with 1.5 gives then the equality

$$
-\operatorname{deg}\left(\Omega_{\bar{Z}}^{1}\left(\log S_{\bar{Z}}\right)\right)+\operatorname{deg}\left(\check{N}_{\bar{Z} / \overline{\mathcal{M}}}\right)=n \cdot \operatorname{deg}\left(E^{0,2}\right)=-n \cdot \operatorname{deg}\left(E^{2,0}\right),
$$

as claimed in i) and ii).

For the Addendum we use the description of the Higgs bundle of the weight two variation of Hodge structures $\mathbb{W}$ on $\mathcal{M}$ in Remark 1.3. It is the direct sum of two sub Higgs bundles, one in bidegree $(2,0)$ and $(1,1)$, the other in bidegrees $(1,1)$ and $(0,2)$. So $(E, \theta)$ is the sum of $\varphi^{*}(H, \tau)$ and $\varphi^{*}\left(H^{\vee}, \tau^{\vee}\right)$, with $E^{2,0}=\varphi^{*} H^{1,0}$ invertible and with $E^{1,1}=\varphi^{*} H^{0,1} \oplus \varphi^{*} H^{0,1 \vee}$. Here

$$
E_{\diamond}^{1,1} \otimes E^{2,0^{-1}}=\varphi^{*}\left(H^{0,1} \otimes H^{1,0^{-1}}\right) \cong \varphi^{*} T_{\overline{\mathcal{M}}}\left(-\log S_{\overline{\mathcal{M}}}\right) .
$$

Since $(H, \tau)$ is the Higgs bundle of a local systems on $\mathcal{M}$, its first Chern class is zero. The rank of $H^{0,1}$ is $n$ and therefore

$$
\begin{aligned}
\operatorname{deg}\left(\varphi^{*} T_{\overline{\mathcal{M}}}\left(-\log S_{\overline{\mathcal{M}}}\right)\right)=\operatorname{deg}\left(\varphi^{*} H^{0,1}\right)-n \cdot \operatorname{deg}\left(\varphi^{*} H^{1,0}\right)= & \\
-(n+1) \cdot \operatorname{deg}\left(\varphi^{*} H^{1,0}\right) & =-(1+n) \cdot \operatorname{deg}\left(E^{2,0}\right) .
\end{aligned}
$$

The exact sequence (0.7) together with 1.5 implies that

$$
-\operatorname{deg}\left(\Omega_{\bar{Z}}^{1}\left(\log S_{\bar{Z}}\right)\right)+\operatorname{deg}\left(\check{N}_{\bar{Z} / \bar{M}}\right)=-(n+1) \cdot \operatorname{deg}\left(E^{2,0}\right),
$$

hence the left hand equality in the Addendum 2.4.

The method to obtain the inequality and the interpretation of the extremal case is parallel to the one used in [STZ03] for the case $\operatorname{dim}(Z)=1$ :

i) Consider the largest saturated Higgs subbundle $(F, \theta)$ of $(E, \theta)$ containing $E^{2,0}$. Hence writing as in [VZ05, Definition 1.7] $\mathrm{Im}^{\prime}$ for the saturated image, we get

$$
(F, \theta)=\left(E^{2,0} \oplus \operatorname{Im}^{\prime}\left(E^{2,0} \otimes T_{\bar{Z}}\left(-\log S_{\bar{Z}}\right)\right) \oplus \operatorname{Im}^{\prime}\left(E^{2,0} \otimes S^{2} T_{\bar{Z}}\left(-\log S_{\bar{Z}}\right)\right), \theta\right) .
$$

The description of the Higgs bundle in Lemma $2.2 \mathrm{~b}$ ) implies that the saturated image $\operatorname{Im}^{\prime}\left(E^{2,0} \otimes S^{2} T_{\bar{Z}}\left(-\log S_{\bar{Z}}\right)\right)$ is non-zero, hence it is isomorphic to $E^{0,2}$. 
By Simpson [Si92] $(E, \theta)$ is a $\mu$-polystable Higgs bundle and therefore

$$
\begin{aligned}
\operatorname{deg}\left(E^{2,0}\right)+\operatorname{deg}\left(\operatorname { I m } ^ { \prime } \left(E^{2,0}\right.\right. & \left.\left.\otimes T_{\bar{Z}}\left(-\log S_{\bar{Z}}\right)\right)\right) \\
& +\operatorname{deg}\left(\operatorname{Im}^{\prime}\left(E^{2,0} \otimes S^{2} T_{\bar{Z}}\left(-\log S_{\bar{Z}}\right)\right)\right)=\operatorname{deg}(F) \leq 0 .
\end{aligned}
$$

Since $F^{2,0}=E^{2,0}$ and $F^{0,2}=E^{0,2}$ are dual to each other $\operatorname{deg}\left(F^{1,1}\right)=\operatorname{deg}(F) \leq 0$.

The morphism $\varphi: Z \rightarrow \mathcal{M}$ is generically finite over its image, hence the natural inclusion $T_{\bar{Z}}\left(-\log S_{\bar{Z}}\right) \rightarrow \varphi^{*} T_{\overline{\mathcal{M}}}\left(-\log S_{\overline{\mathcal{M}}}\right)$ is injective and

$$
\theta: E^{2,0} \otimes T_{\bar{Z}}\left(-\log S_{\bar{Z}}\right) \longleftrightarrow \operatorname{Im}^{\prime}\left(E^{2,0} \otimes T_{\bar{Z}}\left(-\log S_{\bar{Z}}\right)\right)
$$

is an isomorphism over some open dense subscheme. Since $\omega_{\bar{Z}}\left(S_{\bar{Z}}\right)$ is nef, this implies that $\operatorname{deg}\left(E^{2,0} \otimes T_{\bar{Z}}\left(-\log S_{\bar{Z}}\right)\right) \leq \operatorname{deg} F^{1,1} \leq 0$. From this we obtain the Arakelov inequality

$$
\operatorname{deg}\left(E^{2,0}\right) \leq-\mu\left(T_{\bar{Z}}\left(-\log S_{\bar{Z}}\right)\right)=\frac{\operatorname{deg}\left(\Omega_{\bar{Z}}^{1}\left(\log S_{\bar{Z}}\right)\right)}{d}
$$

stated in i). Assume now, that this is an equality. Since $\omega_{\bar{Z}}\left(S_{\bar{Z}}\right)$ is nef and ample with respect to $Z$, this forces the inclusion in (2.2) to be an isomorphism on $Z$. In particular the two sheaves

$$
E^{2,0} \otimes T_{\bar{Z}}\left(-\log S_{\bar{Z}}\right) \quad \text { and } \quad F^{1,1}=\operatorname{Im}^{\prime}\left(E^{2,0} \otimes T_{\bar{Z}}\left(-\log S_{\bar{Z}}\right)\right)
$$

are $\mu$-equivalent, as defined in [VZ05, Definition 1.7].

This equality also implies that

$$
\operatorname{deg}\left(E^{0,2}\right)=\operatorname{deg}\left(\operatorname{Im}^{\prime}\left(E^{2,0} \otimes S^{2} T_{\bar{Z}}\left(-\log S_{\bar{Z}}\right)\right)\right)=\operatorname{deg}\left(E^{2,0}\right)+2 \mu\left(T_{\bar{Z}}\left(-\log S_{\bar{Z}}\right)\right) .
$$

By Yau's Uniformization Theorem [Ya93] the sheaf $S^{2} \Omega_{\bar{Z}}^{1}\left(\log S_{\bar{Z}}\right)$ is $\mu$-polystable, and hence the saturated image of

$$
E^{2,0} \otimes S^{2} T_{\bar{Z}}\left(-\log S_{\bar{Z}}\right) \longrightarrow E^{0,2}
$$

has to be $\mu$-equivalent to one of the direct factors. Again, the ampleness of $\omega_{\bar{Z}}\left(S_{\bar{Z}}\right)$ with respect to $Z$ implies that the morphism in (2.3) is surjective over $Z$.

By [VZ05, Proposition 2.4] we are allowed to apply Simpson's Higgs polystability, proven in [Si92], although the slopes are taken with respect to a non-ample invertible sheaf. Since $F \subset E$ is $\mu$-equivalent to its saturated image and of degree zero, since $E^{2,0}=F^{2,0}$ and $E^{0,2}=F^{0,2}$, one gets a direct sum decomposition

$$
(E, \theta)=(F, \theta) \oplus\left(U^{1,1}, 0\right)
$$


of Higgs bundles. The orthogonality of the splitting with respect to the Hodge metric implies that $\left(U^{1,1}, 0\right)$ comes from a unitary local system. By $1.5 Z$ is a subvariety of $\mathrm{SO}(d, 2)$ of Hodge type.

ii) The proof is similar. Here the saturated Higgs subsheaf $(F, \theta)$ generated by $E^{2,0}$ is given by

$$
(F, \theta)=\left(E^{2,0} \oplus \operatorname{Im}^{\prime}\left(E^{2,0} \otimes T_{\bar{Z}}\left(-\log S_{\bar{Z}}\right)\right), \theta\right) .
$$

Then

$$
\operatorname{deg}\left(E^{2,0}\right)+\operatorname{deg}\left(\operatorname{Im}^{\prime}\left(E^{2,0} \otimes T_{\bar{Z}}\left(-\log S_{\bar{Z}}\right)\right)\right) \leq 0
$$

and the corresponding Arakelov inequality says

$$
\operatorname{deg} E^{2,0} \leq \frac{\operatorname{deg} \Omega_{\bar{Z}}^{1}\left(\log S_{\bar{Z}}\right)}{d+1} .
$$

The equality holds if and only there is a decomposition

$$
(E, \theta)=(F, \theta) \oplus(F, \theta)^{\vee} \oplus\left(U^{1,1}, 0\right),
$$

such that

$$
\theta: E^{2,0} \otimes T_{\bar{Z}}\left(-\log S_{\bar{Z}}\right) \rightarrow \operatorname{Im}^{\prime}\left(E^{2,0} \otimes T_{\bar{Z}}\left(-\log S_{\bar{Z}}\right)\right)
$$

is an isomorphism over $Z$, hence a $\mu$-equivalence. Again $\left(U^{1,1}, 0\right)$ is the Higgs bundle of a unitary local system in this case, and by $1.5 Z$ is a Shimura subvariety of Hodge type for $\mathrm{SU}(d, 1)$ if $d \geq 2$ or a deformation of such for $d=1$.

iii) Finally let $\mathcal{M}$ be a Shimura variety of $\mathrm{SU}(n, 1)$-type. Using the notation from Remark 1.3 the uniformizing Higgs bundle of weight one has the Higgs field

$$
\tau: T_{\mathcal{M}} \otimes H^{1,0} \stackrel{\simeq}{\longrightarrow} H^{0,1} .
$$

In [VZ05] we proved the Arakelov inequality, saying that

$$
(d+1) \cdot \operatorname{deg} E^{2,0}=(d+1) \cdot \operatorname{deg}\left(\varphi^{*}\left(H^{1,0}\right)\right) \leq \operatorname{deg}\left(\Omega_{\bar{Z}}^{1}\left(\log S_{\bar{Z}}\right)\right),
$$

and that the equality forces $Z$ to be a Shimura subvariety of Hodge type for $\mathrm{SU}(d, 1)$. In the present situation the proof is quite simple. Let $\left(H_{\bar{Z}}, \tau_{\bar{Z}}\right)$ denote the Higgs field on $\bar{Z}$ induced by the Deligne extension. Take the sub Higgs sheaf $(F, \theta)$ generated by $H_{\bar{Z}}^{1,0}$. Again Simpson's Higgs-polystability shows that the degree of

$$
(F, \theta)=\left(E^{1,0} \oplus \operatorname{Im}^{\prime}\left(T_{\bar{Z}}\left(-\log S_{\bar{Z}}\right) \otimes E^{1,0 \vee}\right), \theta\right)
$$

is non-positive, hence that (2.4) holds. 
The equality implies that $Z \subset \mathcal{M}$ is totally geodesic. Since $\mathcal{M}$ is of type $\mathrm{SU}(n, 1)$ the sheaf $\Omega_{\overline{\mathcal{M}}}^{1}\left(\log S_{\overline{\mathcal{M}}}\right)$ is ample with respect to $\mathcal{M}$. Then the subvariety $Z \subset \mathcal{M}$ is rigid. Hence by Theorem $1.1 Z$ is a Shimura subvariety of Hodge type for $\operatorname{SU}(d, 1)$.

Remark 2.5. We say that the Hirzebruch-Höfer proportionality (HHP) holds, if the inequalities

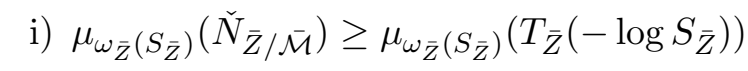

ii) $(d+1) \cdot \operatorname{deg}_{\omega_{\bar{Z}}\left(S_{\bar{Z}}\right)}\left(\check{N}_{\bar{Z} / \overline{\mathcal{M}}}\right) \geq(n-d-1) \cdot \operatorname{deg}_{\omega_{\bar{Z}}\left(S_{\bar{Z}}\right)}\left(T_{\bar{Z}}\left(-\log S_{\bar{Z}}\right)\right)$

iii) $(d+1) \cdot \operatorname{deg}_{\omega_{\bar{Z}}\left(S_{\bar{Z}}\right)}\left(\check{N}_{\bar{Z} / \overline{\mathcal{M}}}\right) \geq(n-d) \cdot \operatorname{deg}_{\omega_{\bar{Z}}\left(S_{\bar{Z}}\right)}\left(T_{\bar{Z}}\left(-\log S_{\bar{Z}}\right)\right)$

in Theorem 2.3 i), ii) and in the Addendum 2.4 iii) are equalities.

If $Z$ is a divisor in $\mathcal{M}$, hence $n=d+1$, then the HHP in Theorem 2.3, ii), just says that the degree of the logarithmic normal sheaf is non-negative, and that $Z$ is a Shimura subvariety of Hodge type, if and only if it is zero.

Proof of Theorem 0.1. For $\bar{Y}=\overline{\mathcal{M}}$ and for a non-singular curve $\bar{C}=\bar{Z} \subset \bar{Y}$ the equality of Chern numbers $(0.8) \operatorname{gives} \operatorname{deg}\left(\check{N}_{\bar{C} / \bar{Y}}\right)=\bar{C} \cdot \bar{C}+S_{\bar{C}}-S_{\bar{Y}} \cdot \bar{C}$. Since $n=2$ and $d=1$ the inequality i) in 2.5 says that

$$
\bar{C} \cdot \bar{C}+\operatorname{deg}\left(S_{\bar{C}}\right)-S_{\bar{Y}} \cdot \bar{C} \geq \operatorname{deg}\left(-K_{\bar{C}}-S_{\bar{C}}\right)=\left(-K_{\bar{Y}}-\bar{C}\right) \cdot \bar{C}-\operatorname{deg}\left(S_{\bar{C}}\right),
$$

as stated in (0.1). The inequality iii) translates to

$$
2 \cdot \bar{C} \cdot \bar{C}+2 \cdot \operatorname{deg}\left(S_{\bar{C}}\right)-2 \cdot S_{\bar{Y}} \cdot \bar{C} \geq\left(-K_{\bar{Y}}-\bar{C}\right) \cdot \bar{C}-\operatorname{deg}\left(S_{\bar{C}}\right),
$$

hence to $(0.2)$.

The remaining inequality ii) is the additional inequality (0.3). However, as explained in the introduction, the assumptions made for ii) imply that $Y$ is the product of two curves and $C$ one of the fibres, so $\bar{C} \cdot \bar{C}=0$.

\section{Subvarieties of $\mathcal{M}$ Containing Special subvarieties}

From now on $\mathcal{M}$ will be a Shimura variety of type $\mathrm{SO}(n, 2)$. We consider a closed subvariety $Z \subset \mathcal{M}$, and we study subvarieties $W \subset Z$ which are Shimura subvarieties of $\mathcal{M}$ of Hodge type. We hope that the existence of sufficiently many 
of them forces $Z$ to be itself a Shimura subvariety of Hodge type. In Section 4 we will see, that this hope is fulfilled if their codimension in $Z$ is one.

Assumptions 3.1. Consider a projective manifold $\bar{Z}$ and the complement $Z$ of a strict normal crossing divisor $S_{\bar{Z}}$. Assume one has generically finite morphisms

$$
W \stackrel{\psi}{\longrightarrow} Z \stackrel{\varphi}{\longrightarrow} \mathcal{M} \text { and } \sigma=\varphi \circ \psi,
$$

such that $\sigma(W)$ is not contained in the singular locus of $\varphi(Z)$. We write

$$
n=\operatorname{dim}(\mathcal{M}), \quad d=\operatorname{dim}(Z) \quad \text { and } \quad m=\operatorname{dim}(W) .
$$

Assume that $\mathcal{M}$ is a Shimura variety of type $\mathrm{SO}(n, 2)$, that $W$ is the deformation of a Shimura subvariety of Hodge type, and that $\sigma$ is induced by a morphism of groups. In particular its image is non-singular and $\sigma$ is étale over the image. We choose Mumford compactifications $\overline{\mathcal{M}}=\mathcal{M} \cup S_{\overline{\mathcal{M}}}$ and $\bar{W}=W \cup S_{\bar{W}}$ and write again

$$
\bar{W} \stackrel{\psi}{\longrightarrow} \bar{Z} \stackrel{\varphi}{\longrightarrow} \overline{\mathcal{M}}
$$

for the induced rational maps. We keep the assumption that the uniformizing variation of Hodge structures $\mathbb{V}$ on $\mathcal{M}$ has unipotent local monodromy at infinity, and we decompose $\mathbb{V}$ as a direct sum $\mathbb{W} \oplus \mathbb{U}$, where $\mathbb{U}$ is the largest unitary subvariation of Hodge structures.

$$
\left(E_{\bar{W}}, \theta_{\bar{W}}\right), \quad\left(E_{\bar{Z}}, \theta_{\bar{Z}}\right) \quad \text { and } \quad\left(E_{\overline{\mathcal{M}}}, \theta_{\overline{\mathcal{M}}}\right)
$$

denote the Higgs bundles induced by the Deligne extension of $\sigma^{*} \mathbb{W}, \varphi^{*} \mathbb{W}$ and $\mathbb{W}$.

Recall that by 0.4 the pullbacks under rational maps are just the reflexive hulls of the pullback to the largest open subscheme, where the morphisms are defined. In particular one has on $\bar{W}$ the natural maps

$$
T_{\bar{W}}\left(-\log S_{\bar{W}}\right) \longrightarrow \psi^{*} T_{\bar{Z}}\left(-\log S_{\bar{Z}}\right) \longrightarrow \sigma^{*} T_{\overline{\mathcal{M}}}\left(-\log S_{\overline{\mathcal{M}}}\right)
$$

By Lemma 2.2 the sheaf $\sigma^{*} T_{\overline{\mathcal{M}}}\left(-\log S_{\overline{\mathcal{M}}}\right)$ is locally free, whereas $\psi^{*} T_{\bar{Z}}\left(-\log S_{\bar{Z}}\right)$ is just torsion-free. Since $\sigma(W)$ meets the non-singular locus of $\varphi(Z)$ both morphisms in (3.1) are injective. We define again the logarithmic normal sheaf by the exact sequence

$$
0 \longrightarrow T_{\bar{W}}\left(-\log S_{\bar{W}}\right) \longrightarrow \psi^{*} T_{\bar{Z}}\left(-\log S_{\bar{Z}}\right) \longrightarrow \check{N}_{\bar{W} / \bar{Z}} \longrightarrow 0 .
$$

\section{Lemma 3.2.}

a. The sheaf $\check{N}_{\bar{W} / \bar{Z}}$ is torsion-free and the exact sequence (3.2) splits. 
b. Let $\check{N}_{\bar{W} / \bar{Z}}^{\natural}$ be the saturated hull of $\check{N}_{\bar{W} / \bar{Z}}$ in $\sigma^{*} T_{\overline{\mathcal{M}}}\left(-\log S_{\overline{\mathcal{M}}}\right)$, i.e.

$$
\begin{aligned}
& \check{N}_{\bar{W} / \bar{Z}}^{\natural}=\operatorname{Ker}\left[\sigma^{*} T_{\overline{\mathcal{M}}}\left(-\log S_{\overline{\mathcal{M}}}\right) \rightarrow\left(\sigma^{*} T_{\overline{\mathcal{M}}}\left(-\log S_{\overline{\mathcal{M}}}\right) /_{\check{N}_{\bar{W} / \bar{Z}}}\right) / \text { torsion }\right] . \\
& \text { Then } \mu_{\omega_{\bar{W}}\left(S_{\bar{W}}\right)}(\mathcal{F})\left(\check{N}_{\bar{W} / \bar{Z}}\right) \leq \mu_{\omega_{\bar{W}}\left(S_{\bar{W}}\right)}\left(\check{N}_{\bar{W} / \bar{Z}}^{\natural}\right) .
\end{aligned}
$$

Proof. Since $\sigma: W \rightarrow \mathcal{M}$ maps to a Shimura subvariety of Hodge type, or to a deformation of such a variety, we are allowed to apply Lemma $2.2 \mathrm{~d}$ ). So there is a surjection $\eta: \sigma^{*} T_{\overline{\mathcal{M}}}\left(-\log S_{\overline{\mathcal{M}}}\right) \rightarrow T_{\bar{W}}\left(-\log S_{\bar{W}}\right)$ whose restriction to the subsheaf $T_{\bar{W}}\left(-\log S_{\bar{W}}\right)$ is an isomorphism. So the restriction of $\eta$ to $\psi^{*} T_{\bar{Z}}\left(-\log S_{\bar{Z}}\right)$ defines a splitting of this sheaf as well.

The sheaf $\check{N}_{\bar{W} / \bar{Z}}$ is contained in $\check{N}_{\bar{W} / \overline{\mathcal{M}}}$ and by Lemma 2.2 the latter is locally free. Part b) follows since $\omega_{\bar{W}}\left(S_{\bar{W}}\right)$ is nef.

Theorem 3.3. Under the Assumptions made in 3.1 one has:

i) If the Griffiths-Yukawa coupling is non-zero on $W$, then

$$
\mu_{\omega_{\bar{W}}\left(S_{\bar{W}}\right)}\left(\check{N}_{\bar{W} / \bar{Z}}^{\natural}\right) \leq \mu_{\omega_{\bar{W}}\left(S_{\bar{W}}\right)}\left(T_{\bar{W}}\left(-\log S_{\bar{W}}\right)\right)<0 .
$$

ii) If the Griffiths-Yukawa coupling on $Z$ vanishes, then

$$
\frac{\operatorname{deg}_{\omega_{\bar{W}}\left(S_{\bar{W})}\right)}\left(\check{N}_{\bar{W} / \bar{Z}}^{\natural}\right)}{\operatorname{rk} \bar{N}_{\bar{W} / \bar{Z}}} \leq \frac{\operatorname{deg}_{\omega_{\bar{W}}\left(S_{\bar{W}}\right)}\left(T_{\bar{W}}\left(-\log S_{\bar{W}}\right)\right)}{m+1}<0 .
$$

iii) Assume that $W \rightarrow \mathcal{M}$ is the deformation of a Shimura curve of Hodge type, and that $Z \subset \mathcal{M}$ is a quasi-projective surface. Then

$$
\operatorname{deg}_{\omega_{\bar{W}}\left(S_{\bar{W}}\right)} \check{N}_{\bar{W} / \bar{Z}}^{\natural} \leq 0 \text {. }
$$

If this is an equality, then the Griffiths-Yukawa coupling along $W$ vanishes and the Griffiths-Yukawa coupling on $Z$ does not vanish.

In order to state what happens if the inequalities in Theorem 3.3 are equalities, we need some more notations. Recall that the Higgs field

$$
\theta: T_{\overline{\mathcal{M}}}\left(-\log S_{\overline{\mathcal{M}}}\right) \otimes E_{\overline{\mathcal{M}}}^{2,0} \longrightarrow E_{\overline{\mathcal{M}}}^{1,1}
$$

is an isomorphism. Consider the tautological sequence for $\varphi: \bar{Z} \rightarrow \overline{\mathcal{M}}$

$$
0 \longrightarrow T_{\bar{Z}}\left(-\log S_{\bar{Z}}\right) \longrightarrow \varphi^{*} T_{\overline{\mathcal{M}}}\left(-\log S_{\overline{\mathcal{M}}}\right) \longrightarrow \check{N}_{\bar{Z} / \overline{\mathcal{M}}} \longrightarrow 0 .
$$

Via the identification $T_{\overline{\mathcal{M}}}\left(-\log S_{\overline{\mathcal{M}}}\right) \otimes E_{\overline{\mathcal{M}}}^{2,0}=E_{\overline{\mathcal{M}}}^{1,1}$ the inclusion

$$
T_{\bar{Z}}\left(-\log S_{\bar{Z}}\right) \longrightarrow \varphi^{*} T_{\overline{\mathcal{M}}}\left(-\log S_{\overline{\mathcal{M}}}\right)
$$


tensorized with $\operatorname{id}_{E_{\bar{Z}}^{2,0}}$ is $\theta: T_{\bar{Z}}\left(-\log S_{\bar{Z}}\right) \otimes E_{\bar{Z}}^{2,0} \longrightarrow E_{\bar{Z}}^{1,1}$.

We now consider the saturated Higgs subsheaf $(F, \theta) \subset\left(E_{\bar{Z}}, \theta_{\bar{Z}}\right)$, which is generated by $E_{\bar{Z}}^{2,0}$. So one has

$$
\begin{gathered}
F^{2,0}=E_{\bar{Z}}^{2,0}, \quad F^{1,1}=\operatorname{Im}^{\prime}\left(\theta: T_{\bar{Z}}\left(-\log S_{\bar{Z}}\right) \rightarrow E_{\bar{Z}}^{1,1}\right), \\
\text { and } \quad F^{0,2}=\operatorname{Im}^{\prime}\left(\theta^{2}: S^{2} T_{\bar{Z}}\left(-\log S_{\bar{Z}}\right) \rightarrow E_{\bar{Z}}^{0,2}\right) .
\end{gathered}
$$

In particular $F^{0,2}$ is zero if the Griffiths-Yukawa coupling is zero, and equal to $E^{0,2}$ otherwise.

Addendum 3.4. In Theorem 3.3 assume that:

$$
\begin{aligned}
& \left.\mu_{\omega_{\bar{W}}\left(S_{\bar{W}}\right)}\left(\check{N}_{\bar{W} / \bar{Z}}\right)=\mu_{\omega_{\bar{W}}\left(S_{\bar{W}}\right)}\left(T_{\bar{W}}\left(-\log S_{\bar{W}}\right)\right) \quad \text { in case } i\right),
\end{aligned}
$$

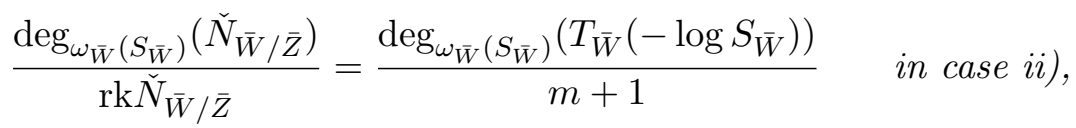

$$
\begin{aligned}
& \operatorname{deg}_{\omega_{\bar{W}}\left(S_{\bar{W}}\right)} \check{N}_{\bar{W} / \bar{Z}}=0 \quad \text { in case } \text { iii). }
\end{aligned}
$$

Then $\psi^{*} \operatorname{Im}\left(\theta: T_{\bar{Z}}\left(-\log S_{\bar{Z}}\right) \rightarrow E_{\bar{Z}}^{1,1}\right)$ and $\psi^{*} F^{1,1}$ are torsionfree. The inclusions

$$
\psi^{*} \operatorname{Im}\left(\theta: T_{\bar{Z}}\left(-\log S_{\bar{Z}}\right) \rightarrow E_{\bar{Z}}^{1,1}\right) \longrightarrow \psi^{*} F^{1,1} \longrightarrow\left(\psi^{*} F^{1,1}\right)^{\natural}
$$

are isomorphisms on $W$ and $\mu_{\omega_{\bar{W}}\left(S_{\bar{W}}\right)}$ equivalences.

Moreover $\left(\psi^{*} F\right)^{\natural}$ is a direct factor of $E_{\bar{W}}$, hence locally free and $\mathrm{c}_{1}\left(\left(\psi^{*} F\right)^{\natural}\right)$ is zero. In particular on $W$ the sequence

$$
0 \longrightarrow \psi^{*} T_{Z} \longrightarrow \sigma^{*} T_{\mathcal{M}} \longrightarrow \psi^{*} N_{Z / \mathcal{M}} \longrightarrow 0
$$

remains exact and splits.

Recall that an inclusion $\mathcal{F} \subset \mathcal{G}$ is a $\mu_{\omega_{\bar{W}}\left(S_{\bar{W}}\right)}$-equivalence, if both sheaves have the same rank, and if $\mu_{\omega_{\bar{W}}\left(S_{\bar{W}}\right)}(\mathcal{F})=\mu_{\omega_{\bar{W}}\left(S_{\bar{W}}\right)}(\mathcal{G})$. Since $\omega_{\bar{W}}\left(S_{\bar{W}}\right)$ is nef and ample with respect to $W$ this implies that $\mathcal{F} \rightarrow \mathcal{G}$ is an isomorphism over $W$.

The statement of Addendum 3.4 says that infinitesimally in a neighborhood of $W$ the subscheme $Z$ of $\mathcal{M}$ looks like a Shimura subvariety. In the next section we will show that such an information for sufficiently many divisors forces $Z$ to be a Shimura variety.

Proof of Theorem 3.3 and of the Addendum 3.4.

For simplicity from now on slopes and degrees will always be with respect to 
$\mathrm{c}_{1}\left(\omega_{\bar{W}}\left(S_{\bar{W}}\right)\right)$, so we drop the lower index and write $\operatorname{deg}(\mathcal{F})$ and $\mu(\mathcal{F})$ instead of $\operatorname{deg}_{\omega_{\bar{W}}\left(S_{\bar{W}}\right)}(\mathcal{F})$ and $\mu_{\omega_{\bar{W}}\left(S_{\bar{W}}\right)}(\mathcal{F})$.

We will also write $(E, \theta)$ instead of $\left(E_{\bar{W}}, \theta_{\bar{W}}\right)$ for the Higgs field induced by the Deligne extension of the variation of Hodge structures to $\bar{W}$. In order to prove Theorem 3.3 and Addendum 3.4 we will consider each of the cases i), ii) and iii) separately.

Let us start with case i). Since $W$ is a subvariety of Hodge type and since the Griffiths-Yukawa coupling is non-zero, there is a decomposition of the form

$$
\left.\left(E^{2,0} \oplus E^{1,1} \oplus E^{0,2}, \theta\right)\right|_{W}=\left.\left.\left(E^{2,0} \oplus E_{\diamond}^{1,1} \oplus E^{0,2}, \theta\right)\right|_{W} \oplus\left(U^{1,1}, 0\right)\right|_{W},
$$

such that the first component uniformizes $W$ and such that the second component is the Higgs field of a unitary variation of Hodge structures of bidegree $(1,1)$. The compatibility of the Deligne extension with pullbacks implies that this decomposition extends to $\bar{W}$.

Since $E_{\diamond}^{1,1} \simeq T_{\bar{W}}\left(-\log S_{\bar{W}}\right) \otimes E^{2,0}$ is $\omega_{\bar{W}}\left(S_{\bar{W}}\right)$-polystable of slope zero and since $U^{1,1}$ corresponds to a unitary local system over $\bar{W}$, we see that

$\sigma^{*} T_{\overline{\mathcal{M}}}\left(-\log S_{\overline{\mathcal{M}}}\right) \simeq E^{1,1} \otimes E^{0,2}=\left(E_{\diamond}^{1,1} \oplus U^{1,1}\right) \otimes E^{0,2} \simeq T_{\bar{W}}\left(-\log S_{\bar{W}}\right) \oplus U^{1,1} \otimes E^{0,2}$ is $\omega_{\bar{W}}\left(S_{\bar{W}}\right)$-polystable and that $T_{\bar{W}}\left(-\log S_{\bar{W}}\right)$ is a direct factor of the sheaf $\sigma^{*} T_{\overline{\mathcal{M}}}\left(-\log S_{\overline{\mathcal{M}}}\right)$, hence of $\psi^{*} T_{\bar{Z}}\left(-\log S_{\bar{Z}}\right)$.

By the exact sequence $(3.2)$ the projection from $\sigma^{*} T_{\overline{\mathcal{M}}}\left(-\log S_{\overline{\mathcal{M}}}\right)$ to $U^{1,1} \otimes E^{0,2}$ induces an injection

$$
\check{N}_{\bar{W} / \bar{Z}}^{\natural} \longrightarrow U^{1,1} \otimes E^{0,2}
$$

So

$$
\mu\left(\check{N}_{\bar{W} / \bar{Z}}^{\natural}\right) \leq \mu\left(U^{1,1} \otimes E^{0,2}\right)=\mu\left(T_{\bar{W}}\left(-\log S_{\bar{W}}\right)\right),
$$

as stated in Part i).

By Lemma 3.2 the sheaf $F^{1,1}$ is without torsion. Since

$$
\mu\left(\check{N}_{\bar{W} / \bar{Z}}\right) \leq \mu\left(\check{N}_{\bar{W} / \bar{Z}}^{\natural}\right) \leq \mu\left(U^{1,1} \otimes E^{0,2}\right),
$$

the equality (3.5) shows that $\check{N}_{\bar{W} / \bar{Z}}$ and $\check{N}_{\bar{W} / \bar{Z}}^{\natural}$ are $\mu$-equivalent. Moreover, as explained in [VZ05, Proposition 2.4] the Simpson correspondence [Si92] implies that $\check{N}_{\bar{W} / \bar{Z}}^{\natural} \otimes E^{2,0}$ is a direct factor of $U^{1,1}$. 
The saturated hull $\left(\psi^{*} F^{1,1}\right)^{\natural}$ is nothing but $E_{\diamond}^{1,1} \oplus \check{N}_{\bar{W} / \bar{Z}}^{\natural} \otimes E^{2,0}$, hence of slope zero. Since the Griffiths-Yukawa coupling is non-zero one has $\left(\psi^{*} F^{0,2}\right)=E^{0,2}$, and since $\left(\psi^{*} F^{2,0}\right)=E^{2,0}$ one finds that $\left(\psi^{*} F\right)^{\natural}$ is a direct factor of $E$.

Obviously the three sheaves

$$
\psi^{*} \operatorname{Im}\left(\theta: T_{\bar{Z}}\left(-\log S_{\bar{Z}}\right) \rightarrow E_{\bar{Z}}^{1,1}\right), \quad \psi^{*} F^{1,1} \text { and }\left(\psi^{*} F^{1,1}\right)^{\natural}
$$

are $\mu$-equivalent, so we verified Addendum 3.4 in case i), except of the strict inequality on the right hand side.

Before finishing i), let us consider the case where the Griffiths-Yukawa coupling on $W$ is zero. This holds in iii) by assumption, and in ii) since the Griffiths-Yukawa coupling on $Z$ is zero. Then one obtains on $\bar{W}$ a different type of decomposition,

$$
\left(E^{2,0} \oplus E^{1,1} \oplus E^{0,2}, \theta\right)=\left(E^{2,0} \oplus E_{\diamond}^{1,1}, \theta\right) \oplus\left(U^{1,1}, 0\right) \oplus\left(E_{\diamond}^{1,1 \vee} \oplus E^{0,2}, \theta\right) .
$$

Here $\left(E^{2,0} \oplus E_{\diamond}^{1,1}, \theta\right)$ uniformizes $W$ as a ball quotient, $\left(U^{1,1}, 0\right)$ is the Higgs bundle of a unitary variation of Hodge structures of type $(1,1)$, and $\left(E_{\diamond}^{1,1 \vee} \oplus E^{0,2}, \theta\right)$ is the dual of $\left(E^{2,0} \oplus E_{\diamond}^{1,1}, \theta\right)$.

Note that the uniformization gives $T_{\bar{W}}\left(-\log S_{\bar{W}}\right) \simeq E_{\diamond}^{1,1} \otimes E^{0,2}$. Hence, one has

$$
\begin{gathered}
\sigma^{*} T_{\overline{\mathcal{M}}}\left(-\log S_{\overline{\mathcal{M}}}\right) \simeq E^{1,1} \otimes E^{0,2}=\left(E_{\diamond}^{1,1} \oplus U^{1,1} \oplus E_{\diamond}^{1,1 \vee}\right) \otimes E^{0,2} \\
\simeq T_{\bar{W}}\left(-\log S_{\bar{W}}\right) \oplus U^{1,1} \otimes E^{0,2} \oplus E_{\diamond}^{1,1 \vee} \otimes E^{0,2}
\end{gathered}
$$

and contrary to the case i) $\sigma^{*} T_{\overline{\mathcal{M}}}\left(-\log S_{\overline{\mathcal{M}}}\right)$ is not polystable. Nevertheless the sheaf $T_{\bar{W}}\left(-\log S_{\bar{W}}\right)$ is a direct factor of $\sigma^{*} T_{\overline{\mathcal{M}}}\left(-\log S_{\overline{\mathcal{M}}}\right)$ and of $\psi^{*} T_{\bar{Z}}\left(-\log S_{\bar{Z}}\right)$.

Dividing by $E_{\diamond}^{1,1} \otimes E^{0,2}$ the exact sequence 3.2 defines an embedding

$$
\check{N}_{\bar{W} / \bar{Z}}^{\natural} \hookrightarrow U^{1,1} \otimes E^{0,2} \oplus E_{\diamond}^{1,1 \vee} \otimes E^{0,2}
$$

and $\left(\psi^{*} F^{1,1}\right)^{\natural}=E_{\diamond}^{1,1} \oplus \check{N}_{\bar{W} / \bar{Z}}^{\natural} \otimes E^{2,0}$.

In ii) we assumed that the Griffiths-Yukawa coupling vanishes on $Z$. So the image of $\check{N}_{\bar{W} / \bar{Z}}^{\natural}$ must lie in the kernel of

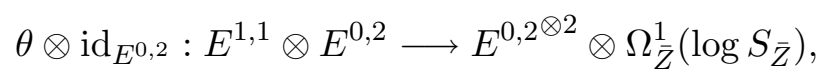

hence in $U^{1,1} \otimes E^{0,2}$. Since the sheaf $U^{1,1} \otimes E^{0,2}$ is $\omega_{\bar{W}}\left(S_{\bar{W}}\right)$-polystable of slope $\operatorname{deg}\left(E^{0,2}\right)$, we obtain

$$
\mu\left(\check{N}_{\bar{W} / \bar{Z}}\right) \leq \mu\left(U^{1,1} \otimes E^{0,2}\right)=\operatorname{deg}\left(E^{0,2}\right) .
$$


Applying Theorem 2.3, ii), to the pair $W \rightarrow \mathcal{M}$ one obtains

$$
\mathrm{c}_{1}\left(T_{\bar{W}}\left(-\log S_{\bar{W}}\right)\right)=(\operatorname{dim}(W)+1) \cdot \mathrm{c}_{1}\left(E^{0,2}\right),
$$

and therefore

$$
\mu\left(\check{N}_{\bar{W} / \bar{Z}}^{\natural}\right) \leq \frac{\operatorname{deg}\left(T_{\bar{W}}\left(-\log S_{\bar{W}}\right)\right)}{\operatorname{dim}(W)+1}
$$

as stated in Part ii).

The equality (3.9) implies that this is an equality and that $\check{N}_{\bar{W} / \bar{Z}} \rightarrow \check{N}_{\bar{W} / \bar{Z}}^{\natural}$ is a $\mu$-equivalence. Moreover one finds

$$
\begin{aligned}
& \operatorname{deg}\left(\left(\psi^{*} F\right)^{\natural}\right)=\operatorname{deg}\left(E^{2,0} \oplus E_{\diamond}^{1,1} \oplus \check{N}_{\bar{W} / \bar{Z}}^{\natural} \otimes E^{2,0}\right)= \\
& (d+1) \cdot \operatorname{deg}\left(E^{2,0}\right)+\operatorname{deg}\left(T_{\bar{W}}\left(-\log S_{\bar{W}}\right)\right)+\operatorname{deg}\left(\check{N}_{\bar{W} / \bar{Z}}^{\natural}\right)= \\
& (d+1-(m+1)-(d-m)) \cdot \operatorname{deg}\left(E^{2,0}\right)=0 .
\end{aligned}
$$

By [VZ05, Proposition 2.4] the Simpson correspondence implies that $\left(\psi^{*} F\right)^{\natural} \subset E$ is a direct factor.

For Theorem 3.3, i) and ii), it remains to verify the strict inequality on the right hand side. In both cases the sheaf $\bar{N}_{\bar{W} / \bar{Z}}^{\natural}$ is isomorphic to a subsheaf of the semistable sheaf $U^{1,1} \otimes E^{0,2}$, hence $\mu\left(\check{N}_{\bar{W} / \bar{Z}}^{\natural}\right) \leq \mu\left(E^{0,2}\right)<0$.

In the remaining case iii) we have again the embedding in (3.8). Since $W$ is a curve, using the notation introduced there, one has

$$
E_{\diamond}^{1,1 \vee} \otimes E^{0,2}=\left(E^{2,0} \otimes T_{\bar{W}}\left(-\log S_{\bar{W}}\right)\right)^{\vee} \otimes E^{0,2}=\mathcal{O}_{\bar{W}}
$$

and $\check{N}_{\bar{W} / \bar{Z}}^{\natural}$ is a subsheaf of $U^{1,1} \otimes T_{\bar{W}}\left(-\log S_{\bar{W}}\right)^{1 / 2} \oplus \mathcal{O}_{\bar{W}}$. This inclusion implies that $\operatorname{deg}\left(\check{N}_{\bar{W} / \bar{Z}}^{\natural}\right) \leq 0$, as stated in Part iii).

If the equation (3.7) holds one has $\check{N}_{\bar{W} / \bar{Z}}=\check{N}_{\bar{W} / \bar{Z}}^{\natural}$ and both are of degree zero. So the projection to the negative sheaf $U^{1,1} \otimes T_{\bar{W}}\left(-\log S_{\bar{W}}\right)^{1 / 2}$ must be zero, hence $\check{N}_{\bar{W} / \bar{Z}}=\mathcal{O}_{\bar{W}}$. Then

$$
\psi^{*} F^{1,1}=T_{\bar{W}}\left(-\log S_{\bar{W}}\right) \otimes E^{2,0} \oplus E^{2,0},
$$

and since $F^{2,0}=E^{2,0}$ and $F^{0,2}=E^{0,2}$ one obtains $\operatorname{deg}\left(\left(\psi^{*} F\right)^{\natural}\right)=0$. Again the Simpson correspondence implies that $\left(\psi^{*} F, \theta\right) \subset(E, \theta)$ is a direct factor. 


\section{A characterization of subvarieties of a Shimura variety $\mathcal{M}$ of TYPE $\mathrm{SO}(n, 2)$.}

In this section we start with an auxiliary result on a finite set of divisors $\left\{Y_{i}\right\}_{i \in I}$ on projective manifolds $X$. Later $X$ will be the compactification of a subscheme of $\mathcal{M}$ and the $Y_{i}$ will be compactifications of Shimura subvarieties $W_{i}$ of $\mathcal{M}$.

Lemma 4.1. Let $X$ be a smooth projective manifold of dimension $d$ and let $\left\{Y_{i}\right\}_{i \in I}$ be a set of pairwise distinct prime divisors. Let $\rho$ be the Picard number of $X$, let $A$ be a nef and big divisor on $X$ and assume that $Y_{i}^{2} \cdot A^{d-2}<0$ for all $i \in I$.

If $\# I \geq \rho^{2}+\rho+1$ then there exists a linear combination $D=\sum_{i \in I} a_{i} Y_{i}$ with $a_{i} \in \mathbb{N}$ and $D^{2} \cdot A^{d-2}>0$.

Proof. Let $\mathrm{NS}(X)_{\mathbb{Q}}$ be the $\mathbb{Q}$-Neron-Severi group of $X$ and let $\equiv$ stand for "numerical equivalence". Remark first that for effective divisors $D$ and $D^{\prime}$ without common components, the intersection $D . D^{\prime}$ is a linear combination of codimension two subschemes with non-negative coefficients. Since $A$ is nef, one obtains $D \cdot D^{\prime} \cdot A^{d-2} \geq 0$.

We start with any subset $I_{1} \subset I$ of cardinality $\rho+1$, say $I_{1}=\{1, \ldots, \rho+1\}$. The images of the divisors $Y_{1}, \ldots, Y_{\rho+1}$ in $\mathrm{NS}(X)_{\mathbb{Q}}$ must be linearly dependent, hence there exist $a_{1}, \ldots, a_{l}, b_{1}, \ldots, b_{m} \in \mathbb{N}$ with

$$
D_{1} \equiv D_{1}^{\prime} \quad \text { for } \quad D_{1}=\sum_{i=1}^{l} a_{i} Y_{i} \quad \text { and } \quad D_{1}^{\prime}=\sum_{j=1}^{m} b_{j} Y_{j} .
$$

Since $D_{1}$ and $D_{1}^{\prime}$ are effective divisors without common components one obtains $D_{1}^{2} \cdot A^{d-2}=D_{1}^{\prime} D_{1} \cdot A^{d-2} \geq 0$. If $D_{1}^{2} \cdot A^{d-2}>0$, we are done.

If $D_{1}^{2} \cdot A^{d-2}=0$ and if there exists a divisor $Y_{j}$ with $j>\rho+1$ and with $D . Y_{j} \cdot A^{d-2}>0$, then for $m$ sufficiently large $\left(m D_{1}+Y_{j}\right)^{2} \cdot A^{d-2}>0$, and again we found the divisor we are looking for.

Hence if the statement of Lemma 4.1 is wrong, for any system of disjoint subsets $I_{1}, \ldots, I_{\rho} \subset I$ with $\# I_{\iota}=\rho+1$, we can find effective non-zero divisors

$$
D_{\iota}=\sum_{i \in I_{\iota}} a_{i} \cdot Y_{i}
$$


with $D_{\iota}^{2} \cdot A^{d-2}=0$ and with $Y_{j} \cdot D_{\iota} \cdot A^{d-2}=0$ for all $j \in I \backslash I_{\iota}$. In particular, choosing $\nu_{\iota} \in I_{\iota}$ with $a_{\nu_{\iota}} \neq 0$ the intersection $Y_{\nu_{\iota}} \cdot Y_{j} \cdot A^{d-2}=0$ for $j \in I \backslash I_{\iota}$.

By assumption $I \backslash\left(I_{1} \cup \cdots \cup I_{\rho}\right)$ still contains one element, say $\nu_{\rho+1}$, and the intersection of $A^{d-2}$ with two different divisors in $\left\{Y_{\nu_{1}}, \ldots, Y_{\nu_{\rho+1}}\right\}$ is zero. So given a linear combination

$$
0=\sum_{\iota=1}^{\rho+1} \alpha_{\iota} \cdot Y_{\nu_{\iota}} \quad \text { one finds } \quad 0=\sum_{\iota=1}^{\rho+1} \alpha_{\iota} \cdot Y_{\nu_{\iota}} \cdot Y_{\nu_{k}} \cdot A^{d-2}=\alpha_{k} \cdot Y_{\nu_{k}} \cdot Y_{\nu_{k}} \cdot A^{d-2},
$$

for all $1 \leq k \leq \rho+1$. The assumption $Y_{i}^{2} \cdot A^{d-2}<0$ implies

$$
\alpha_{1}=\cdots=\alpha_{\rho+1}=0
$$

and hence the images of $\left\{Y_{\nu_{1}}, \ldots, Y_{\nu_{\rho+1}}\right\}$ in $\mathrm{NS}(X)_{\mathbb{Q}}$ are linear independent, a contradiction.

From now on, as indicated in the title of this section, $\mathcal{M}$ will again be a Shimura variety of type $\mathrm{SO}(n, 2)$.

Assumptions 4.2. Consider a projective manifold $\bar{Z}$ of dimension $d \geq 2$ and the complement $Z$ of a strict normal crossing divisor $S_{\bar{Z}}$ with $\Omega_{\bar{Z}}^{1}\left(\log S_{\bar{Z}}\right)$ nef and with $\omega_{\bar{Z}}\left(S_{\bar{Z}}\right)$ ample with respect to $Z$. Given an injection $\varphi: Z \rightarrow \mathcal{M}$ and a finite index set $I$, consider for $i \in I$ non-singular irreducible divisors $W_{i}$ on $Z$, hence $m=\operatorname{dim}\left(W_{i}\right)=d-1$.

The corresponding embeddings will be denoted by

$$
W_{i} \stackrel{\psi_{i}}{\longrightarrow} Z \stackrel{\varphi}{\longrightarrow} \mathcal{M} \text { and } \sigma_{i}=\varphi \circ \psi_{i} .
$$

We assume that $\mathcal{M}$ is a Shimura varieties of type $\mathrm{SO}(n, 2)$, that the $W_{i}$ are deformations of Shimura subvarieties of Hodge type, and that $\sigma_{i}$ is induced by a morphism of groups. Choosing Mumford compactifications $\overline{\mathcal{M}}=\mathcal{M} \cup S_{\overline{\mathcal{M}}}$ and $\bar{W}_{i}=W_{i} \cup S_{\bar{W}_{i}}$, we write again

$$
\bar{W}_{i} \stackrel{\psi_{i}}{\longrightarrow} \bar{Z} \stackrel{\varphi}{\longrightarrow} \overline{\mathcal{M}} \text { and } \sigma_{i}=\varphi \circ \psi_{i}
$$

for the induced rational maps. Let $\mathbb{V}$ be the uniformizing variation of Hodge structures on $\mathcal{M}$, let $\mathbb{U}$ be the largest unitary subvariation of Hodge structures and $\mathbb{V}=\mathbb{W} \oplus \mathbb{U}$. As usual $\left(E_{\bar{Z}}, \theta_{\bar{Z}}\right)$ will denote the Higgs bundle induced by the Deligne extension of $\varphi^{*} \mathbb{W}$. 
Notations 4.3. Let $\rho$ denote the Picard number of $\bar{Z}$ and let $\delta$ denote the number of non-empty intersections $S_{\ell} \cap S_{k}$ of different components $S_{\ell}$ and $S_{k}$ of $S_{\bar{Z}}$. We define $\varsigma(\bar{Z})=\rho^{2}+\rho+1$ if one of the following conditions holds:

(1) $d=\operatorname{dim}(Z) \geq 4$.

(2) For all $i \in I$ the divisor $\left.S_{\bar{Z}}\right|_{\psi_{i}\left(\bar{W}_{i}\right)}-\left(\left.S_{\bar{Z}}\right|_{\psi_{i}\left(\bar{W}_{i}\right)}\right)_{\text {red }}$, considered in (0.8), is zero.

(3) For all $i, j \in I$ one has $\psi_{i}\left(\bar{W}_{i}\right) \cap \psi_{j}\left(\bar{W}_{j}\right) \neq \emptyset$.

Otherwise we choose $\varsigma(\bar{Z})=(\rho+\delta)^{2}+\rho+\delta+1$.

Theorem 4.4. Under the assumptions made in 4.2 one has:

i) If the $W_{i}$ are of type $\mathrm{SO}(d-1,2)$, for all $i \in I$, if they satisfy the $H H P$ equality

$$
\mu_{\omega_{\bar{W}_{i}}\left(S_{\bar{W}_{i}}\right)}\left(\check{N}_{\bar{W}_{i} / \bar{Z}}\right)=\mu_{\omega_{\bar{W}_{i}}\left(S_{\bar{W}_{i}}\right)}\left(T_{\bar{W}_{i}}\left(-\log S_{\bar{W}_{i}}\right)\right),
$$

and if $\# I \geq \varsigma(\bar{Z})$, then $Z \subset \mathcal{M}$ is a Shimura subvariety of Hodge type for $\mathrm{SO}(d, 2)$.

ii) Assume that the Griffiths-Yukawa coupling vanishes on $\bar{Z}$. If the $W_{i}$ are Shimura varieties of type $\mathrm{SU}(d-1,1)$, if

$$
\frac{\operatorname{deg}_{\omega_{\bar{W}_{i}}\left(S_{\bar{W}_{i}}\right)}\left(\check{N}_{\left.\bar{W}_{i} / \bar{Z}\right)}\right.}{\operatorname{rk} \check{N}_{\bar{W}_{i} / \bar{Z}}}=\frac{\operatorname{deg}_{\omega_{\bar{W}_{i}}\left(S_{\bar{W}_{i}}\right)}\left(T_{\bar{W}_{i}}\left(-\log S_{\bar{W}_{i}}\right)\right)}{d+1},
$$

and if $\# I \geq \varsigma(\bar{Z})$, then $Z \subset \mathcal{M}$ is a Shimura subvariety of Hodge type for $\mathrm{SU}(d, 1)$.

iii) Assume that $\bar{Z}$ is a surface and that $I=\{1,2\}$. Assume that

$$
\sigma_{1}\left(\bar{W}_{1}\right) \cap \sigma_{2}\left(\bar{W}_{2}\right) \neq \emptyset
$$

and that $\operatorname{deg} \check{N}_{\bar{W}_{i} / \bar{Z}}=0$. Then $Z$ is the product of two Shimura curves of Hodge type.

Let us start with some preparations for the proof. First of all, by Lemma 1.5 for any coherent sheaf $\mathcal{F}$ one has

$$
\operatorname{deg}_{E^{2,0}}(\mathcal{F})=(d-1)^{d-2} \cdot \operatorname{deg}_{\omega_{\bar{W}}\left(S_{\bar{W}}\right)}(\mathcal{F}) \quad \text { or } \quad \mu_{E^{2,0}}(\mathcal{F})=d^{d-2} \cdot \mu_{\omega_{\bar{W}}\left(S_{\bar{W}}\right)}(\mathcal{F}),
$$

depending on the type of $W_{i}$. 
In both cases we are allowed to replace the slope with respect to $c_{1}\left(\omega_{\bar{W}_{i}}\left(S_{\bar{W}_{i}}\right)\right)$ by the one with respect to $c_{1}\left(E^{2,0}\right)$ or $c_{1}\left(\varphi^{*} \omega_{\overline{\mathcal{M}}}\left(S_{\overline{\mathcal{M}}}\right)\right)$.

The Mumford compactification $\overline{\mathcal{M}}$ maps to the Baily-Borel compactification $\overline{\mathcal{M}}^{*}$, and for $\gamma$ sufficiently large the sheaf $\varphi^{*} \omega_{\overline{\mathcal{M}}}\left(S_{\overline{\mathcal{M}}}\right)^{\gamma}$ is the pullback of a very ample sheaf $\omega_{\overline{\mathcal{M}}^{*}}$ on $\overline{\mathcal{M}}^{*}$ (see [Mu77]). Then the invertible sheaf

$$
\mathcal{L}=\operatorname{det}\left(E_{\bar{Z}}^{1,1} \otimes E_{\bar{Z}}^{2,0}\right)^{-\gamma}=\varphi^{*}\left(\omega_{\overline{\mathcal{M}}}\left(S_{\overline{\mathcal{M}}}\right)\right)^{\gamma}
$$

is semiample. In fact, if $\phi: \hat{Z} \rightarrow \bar{Z}$ is a morphism such that $\bar{Z} \rightarrow \overline{\mathcal{M}}^{*}$ extends to a morphism $\hat{\varphi}: \hat{Z} \rightarrow \overline{\mathcal{M}}^{*}$ the unicity of the Deligne extension implies that

$$
\phi^{*} \varphi^{*} \mathcal{L}=\phi^{*} \operatorname{det}\left(E_{\bar{Z}}^{1,1} \otimes E_{\bar{Z}}^{2,0}\right)^{-\gamma}=\hat{\varphi}^{*} \omega_{\overline{\mathcal{M}}^{*}}^{\gamma},
$$

and hence that $\mathcal{L}$ is generated by global sections. In the same way, one sees that $\psi_{i}^{*} \mathcal{L}$ is an invertible sheaf on $\bar{W}_{i}$ which is generated by global sections.

The dimension of $\overline{\mathcal{M}}^{*} \backslash \mathcal{M}$ is at most one (see [Lo03], for example). Hence given any component $\Delta_{\bar{Z}}$ of the boundary $S_{\bar{Z}}$ (or of $S_{\bar{W}_{i}}$ ) one finds

$$
\begin{gathered}
\Delta . c_{1}(\mathcal{L})^{2}=c_{1}\left(\left.\mathcal{L}\right|_{\Delta}\right)^{2} \equiv 0 \quad\left(\text { or } \quad \Delta . c_{1}\left(\psi_{i}^{*} \mathcal{L}\right)^{2} \equiv 0\right), \quad \text { and } \\
\Delta . c_{1}(\mathcal{L})=c_{1}\left(\left.\mathcal{L}\right|_{\Delta}\right) \equiv 0 \quad\left(\text { or } \quad \Delta . c_{1}\left(\psi_{i}^{*} \mathcal{L}\right) \equiv 0\right),
\end{gathered}
$$

if the dimension of the image of $\Delta_{\bar{Z}}$ or $\Delta_{\bar{W}_{i}}$ in $\overline{\mathcal{M}}^{*}$ is a point.

We will need blowing ups of the Mumford compactification $\mathcal{M}$ such that the proper transform $W_{i}$ meets the boundary transversally outside of codimension two:

Proposition 4.5. For some $i \in I$ let $\Delta$ be a component of $S_{\bar{W}_{i}}$ such that the morphism $\Delta \rightarrow \overline{\mathcal{M}}^{*}$ is finite (hence $d \leq 3$ ). Then there exists a blowing up $\Psi: \overline{\mathcal{M}}_{\Delta} \rightarrow \overline{\mathcal{M}}$, with centers in $S_{\overline{\mathcal{M}}}$, such that:

(1) $S_{\overline{\mathcal{M}}_{\Delta}}=\overline{\mathcal{M}}_{\Delta} \backslash \mathcal{M}$ is a normal crossing divisor and $\Omega_{\overline{\mathcal{M}}_{\Delta}}^{1}\left(\log S_{\overline{\mathcal{M}}_{\Delta}}\right)$ is nef.

(2) In a neighborhood of the general point of $\Delta$ the rational map $\sigma_{i}: W_{i} \rightarrow \mathcal{M}$ extends to an embedding $\sigma_{\Delta}: \bar{W}_{i} \rightarrow \overline{\mathcal{M}}_{\Delta}$ whose image intersects $S_{\overline{\mathcal{M}}_{\Delta}}$ transversally.

Proof. Since the first condition holds on $\overline{\mathcal{M}}$ it will hold on $\overline{\mathcal{M}}_{\Delta}$ if (and only if) we only blow up strata of the boundary divisors. In fact, this is an easy exercise if the center is a point. Since locally along any stratum of $S_{\overline{\mathcal{M}}_{\Delta}}$ the manifold $\overline{\mathcal{M}}$ looks like a product one obtains the general case. 
For the proof one has to compare the toroidal compactifications, constructed in [AMRT75], for two local symmetric domains. Fortunately we will only need this in smooth points of boundary components. A by far more extensive description will be given in Section 2 of the article [And07], and we use this as an excuse, just to sketch the arguments.

Let us fix $i$ and $\Delta$ and drop the lower indices. Recall that $\mathcal{M}=\Gamma \backslash \operatorname{SO}(n, 2) / K$ with $\Gamma$ a neat arithmetic group and that $W=\Gamma^{\prime} \backslash G^{\prime} / K^{\prime}$ is a local symmetric domain. The inclusion $W \rightarrow \mathcal{M}$ is induced by a homomorphism of groups $G^{\prime} \rightarrow$ $G:=\mathrm{SO}(n, 2)$ with a finite kernel. To define the Mumford compactification one needs several data, which we list for $G$. Adding a ' gives the corresponding notations for $G^{\prime}$.

First of all, let $D=G / K \rightarrow D^{\vee}$ be the embedding of $D$ (we drop the ${ }^{+}$, used in the first section) in its compact dual $D^{\vee}$. The maximal analytic submanifolds $F$ of $D^{\vee} \backslash D$ are called the boundary components of $D$. One defines (see [Mu77, $\S 3]):$

- $N(F):=\{g \in G ; g F=F\}$.

- $F$ is rational if $\Gamma \cap N(F)$ is an arithmetic subgroup of $N(F)$.

Recall that the boundary of the Baily-Borel compactification $\overline{\mathcal{M}}^{*} \backslash \mathcal{M}$ is the disjoint union of finitely many subspaces of the form $(\Gamma \cap N(F)) \backslash F$ for rational boundary components $F$. Next we need

- $U(F)=$ the center of the unipotent radical $W(F)$ of $N(F)$, as a vector space $\approx \mathbb{C}^{k}$.

The homomorphism $\tau: G^{\prime} \rightarrow G$ extends to a homomorphism $D^{\prime \vee} \rightarrow D^{\vee}$ (see [AMRT75] and it induces a map from the set of rational boundary components of $D^{\prime}$ to the one of $D$. Moreover, the inclusion $W \subset \mathcal{M}$ extends to a map $\bar{W}^{*} \rightarrow \overline{\mathcal{M}}^{*}$ of the Baily-Borel compactifications, compatible with the map between boundary components. Fixing some boundary component $F^{\prime}$ of $D^{\prime}$ with image $F$, the characterization of boundary components in [AMRT75, III, §3] shows that $F$ is rational if the same holds for $F^{\prime}$. Moreover $\tau$ induces compatible morphisms $N\left(F^{\prime}\right) \rightarrow N(F), W\left(F^{\prime}\right) \rightarrow W(F)$ and $U\left(F^{\prime}\right) \rightarrow U(F)$. Furthermore one needs a self-adjoint open convex cone $C(F) \subset U(F)$, homogeneous under $G$. Again the latter is compatible with $\tau$. The toroidal compactification depends on certain compatible decompositions of the cones $\overline{C(F)}$, for all boundary components. Or, 
if one uses coordinates, as Mumford does in $[\mathrm{Mu} 77, \S 3]$, it is given by a certain basis $\left\{\xi_{1}, \ldots, \xi_{k}\right\}$ of the $\mathbb{Z}$-module $\Gamma \cap U(F)$, with $\xi_{1}, \ldots \xi_{\mu} \in C(F)$ and with $\xi_{\mu+1}, \ldots, \xi_{k} \in \overline{C(F)} \backslash C(F)$, for some $\mu \geq 1$. As we will recall in a moment, each point $q$ in $\overline{\mathcal{M}}$, lying on $S_{\overline{\mathcal{M}}}$ and with image in $(\Gamma \cap N(F)) \backslash F$, has an analytic neighborhood isomorphic an open subset of $\mathbb{C}^{k} \times \mathbb{C}^{\ell} \times F$ with coordinates $\left(z_{1}, \ldots, z_{k}\right)$ on the first factor. Here the intersection with $\mathcal{M}$ corresponds to the intersection with $\mathbb{C}^{* k} \times \mathbb{C}^{\ell} \times F$, and the different boundary components of $S_{\overline{\mathcal{M}}}$ map to the zero sets of $z_{\iota}$ for some $1 \leq \iota \leq \mu$ (see [Mu77, page 256,5)]).

The pullback of the cone decomposition defining $\overline{\mathcal{M}}$ gives a cone decomposition for $F^{\prime}$, hence a second toroidal compactification $\bar{W}^{\prime}$. In a neighborhood of a general point of $\Delta$ we have a morphism $\bar{W} \rightarrow \bar{W}^{\prime}$, and since both map to the Baily-Borel compactification, this morphism will be an embedding. So we may replace $\bar{W}$ by $\bar{W}^{\prime}$. As usual we drop the upper index ', and assume that there is a morphism $\sigma: \bar{W} \rightarrow \mathcal{M}$ of toroidal embeddings.

By assumption, the dimension of $\Delta$ is equal to the one of the rational boundary component $F^{\prime}$, hence in the description given above one has $k^{\prime}=1$ and $\ell^{\prime}=0$, and $U\left(F^{\prime}\right)$ is one dimensional. Hence there is exactly one generator $\xi^{\prime}$ in the cone $C\left(F^{\prime}\right)$. Its image in $C(F)$, again denoted by $\xi^{\prime}$ can be written as a linear combination

$$
\xi^{\prime}=\sum_{i=1}^{k} a_{i} \xi_{i} \quad \text { with } \quad a_{i} \in \mathbb{N} \quad \text { and } \quad \operatorname{ggT}\left\{a_{i} ; a_{i} \neq 0\right\}=1 .
$$

Let us take up the description of local charts, given in [Mu77, page 256]:

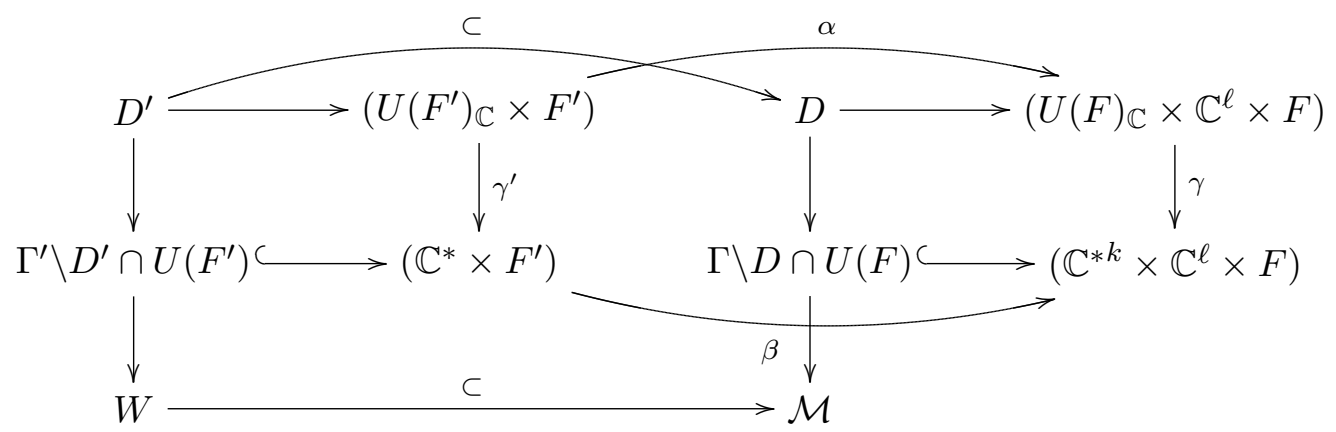

Here using the basis $\left\{\xi_{i}\right\}$ the vectorspace $U(F)_{\mathbb{C}}$ is identified with $\mathbb{C}^{k}$ and

$$
\gamma\left(x_{1}, \ldots, x_{k}\right)=\left(e^{2 \pi \sqrt{-1} \cdot x_{1}}, \ldots, e^{2 \pi \sqrt{-1} \cdot x_{k}}\right),
$$

and the same description holds on the left hand side. 
The morphisms $\alpha$ and $\beta$ respect the product decomposition, and on the first component $\alpha(x)=\left(a_{1} \cdot x, \ldots, a_{k} \cdot x\right)$. So writing the coordinates on $\mathbb{C}^{*}$ and $\mathbb{C}^{* k}$ as $z$ and $\left(z_{1}, \ldots, z_{k}\right)$ one finds $\beta(z)=\left(z^{a_{1}}, \ldots, z^{a_{k}}\right)$, again neglecting the other components.

As above, local neighborhoods of boundary points $q$ of $\overline{\mathcal{M}}$ are given by certain tuples $\left(F,\left\{\xi_{i}\right\}\right)$, and the components of the boundary corresponds to the zero set of some of the first $\mu$ components. We are only interested in those charts, containing the image $p$ of a general point of $\Delta$. So in the description of $\xi^{\prime}$ as a linear combination of the $\xi_{i}$ we can assume that

$$
a_{1} \geq a_{2} \geq \cdots \geq a_{\mu^{\prime}}>a_{\mu^{\prime}+1}=\cdots=a_{k}=0,
$$

for some $1 \leq \mu^{\prime} \leq \mu$. Then the image of each branch of $\bar{W}$ in a neighborhood of $p$ is parameterized by

$$
\beta(z)=\left(z^{a_{1}}, \ldots, z^{a_{\mu^{\prime}}}, 1, \ldots, 1\right) \text {, with } \operatorname{ggT}\left\{a_{1}, \cdots, a_{\mu^{\prime}}\right\}=1 .
$$

If $\mu^{\prime}=1$ we are done. If $\mu^{\prime}>1$ we blow up the corresponding stratum of $S_{\overline{\mathcal{M}}}$. After finitely many steps one finds an embedded resolution such that the proper transform meets the new boundary transversally in the smooth locus.

Corollary 4.6. Let $\Delta$ be an irreducible component of the divisor

$$
\left.S_{\bar{Z}}\right|_{\psi_{i}\left(\bar{W}_{i}\right)}-\left(\left.S_{\bar{Z}}\right|_{\psi_{i}\left(\bar{W}_{i}\right)}\right)_{\mathrm{red}} \in \operatorname{Div}\left(\psi_{i}\left(\bar{W}_{i}\right)\right)
$$

considered in (0.9). Then either $\psi_{i}(\Delta)$ is contained in the intersection $S_{\ell} \cap S_{k}$ of two different components $S_{\ell}$ and $S_{k}$ of $S_{\bar{Z}}$ or $\psi_{i}(\Delta) \cdot c_{1}(\mathcal{L})^{d-2}=0$ for the invertible sheaf $\mathcal{L}$ introduced above.

Proof. We will assume that $\psi_{i}(\Delta)$ is just contained in one component $S_{\ell}$, and we will show, that its multiplicity in $\left.S_{\bar{Z}}\right|_{\psi_{i}\left(\bar{W}_{i}\right)}$ is at most one. To this aim, we use Proposition 4.5 to choose the blowing up $\overline{\mathcal{M}}_{\Delta}$ of the given Mumford compactification. By abuse of notations we drop the indices $i$ and ${ }_{\Delta}$.

In order to verify the Corollary 4.6, we also may replace $Z$ by the intersection with $d-2$ general divisors $L_{1}, \ldots, L_{d-2}$ of the invertible sheaf $\mathcal{L}$, introduced above, and correspondingly $\bar{W}$ by the intersection of $\psi^{*} L_{1}, \ldots, \psi^{*} L_{d-2}$. In fact, if $\Delta$ does not meet this intersection, there is nothing to show. In particular, as remarked in (4.1) this intersection will be trivial if the fibres of $\Delta \rightarrow \overline{\mathcal{M}}^{*}$ are positive dimensional. As stated in (4.1) this will always be the case for $d \geq 4$, 
hence $\operatorname{dim}(\Delta) \geq 2$. Remark that the local transversality of the intersection of $\sigma(\bar{W})$ with $S_{\overline{\mathcal{M}}}$ will be preserved under intersection with general $L_{i}$.

So let us restrict ourselves to the case where $\bar{Z}$ is a surface and $\bar{W}$ a curve. We have rational maps

$$
\bar{W} \stackrel{\psi}{\longrightarrow} \bar{Z} \stackrel{\varphi}{\longrightarrow} \overline{\mathcal{M}},
$$

where $\psi$ and $\sigma=\varphi \circ \psi$ are morphisms and where $\Delta$ is a reduced point of $\sigma^{*} S_{\overline{\mathcal{M}}}$. Choose a minimal blowing up $\Phi: \bar{Z}^{\prime} \rightarrow \bar{Z}$ such that the composite $\varphi^{\prime}=\varphi \circ \Phi$ is a morphism. Of course $\psi$ lifts to a morphism $\psi^{\prime}: \bar{W} \rightarrow \bar{Z}^{\prime}$ near $\Delta$. Since

$$
\sigma^{*} S_{\overline{\mathcal{M}}}=\psi^{\prime *} \varphi^{\prime *} S_{\overline{\mathcal{M}}} \geq \psi^{\prime *} S_{\bar{Z}^{\prime}},
$$

the multiplicity of $\Delta$ in $\psi^{\prime *} S_{\bar{Z}^{\prime}}$ is again one and so $\varphi^{\prime *} S_{\overline{\mathcal{M}}}$ is reduced and non singular in a neighborhood of $\psi^{\prime}(\Delta)$. Since $\varphi^{\prime}$ is injective away from the boundary there is a neighborhood of $\psi^{\prime}(\Delta)$ on which the morphism $\varphi^{\prime}$ is an embedding whose image meets $S_{\overline{\mathcal{M}}}$ transversally. So the natural map

$$
\varphi^{\prime *} \Omega_{\overline{\mathcal{M}}}^{1}\left(\log S_{\overline{\mathcal{M}}}\right) \longrightarrow \Omega_{\bar{Z}^{\prime}}^{1}\left(\log S_{\bar{Z}^{\prime}}\right)
$$

will be surjective over this neighborhood. On the other hand, the sheaf on the left hand side is nef, and the same holds true for its image in $\Omega_{\bar{Z}^{\prime}}^{1}\left(\log S_{\bar{Z}^{\prime}}\right)$. So the image has to lie in $\Phi^{*} \Omega_{\bar{Z}}^{1}\left(\log S_{\bar{Z}}\right)$. Since we assumed that $\psi(\Delta)$ is a smooth point of the boundary $S_{\bar{Z}}$, the support of the cokernel of

$$
\Phi^{*} \Omega_{\bar{Z}}^{1}\left(\log S_{\bar{Z}}\right) \subset \Omega_{\bar{Z}^{\prime}}^{1}\left(\log S_{\bar{Z}^{\prime}}\right)
$$

contains the whole exceptional locus. So there is no blowing up, $\varphi$ is a morphism, and $\Delta$ is reduced in $\psi^{*} S_{\bar{Z}}$.

Proof of Theorem 4.4. We start with parts i) and ii).

Let us choose morphisms $\phi_{i}: \hat{W}_{i} \rightarrow \bar{W}_{i}$ such that the rational map $\psi_{i}$ lifts to a morphism $\hat{\psi}_{i}: \hat{W}_{i} \rightarrow \bar{Z}$. Since $\check{N}_{\bar{W}_{i} / \bar{Z}} \hookrightarrow \phi_{i *} \phi_{i}^{*} \check{N}_{\bar{W}_{i} / \bar{Z}} \hookrightarrow \check{N}_{\bar{W}_{i} / \bar{Z}}^{\natural}$ one obtains

$$
\operatorname{deg}_{\hat{\psi}_{i}^{*} \mathcal{L}}\left(\phi_{i}^{*} \check{N}_{\bar{W}_{i} / \bar{Z} / \text { torsion }}\right)=\operatorname{deg}_{\psi_{i}^{*} \mathcal{L}}\left(\phi_{i_{*}} \phi_{i}^{*} \check{N}_{\bar{W}_{i} / \bar{Z}}\right)=\operatorname{deg}\left(\check{N}_{\bar{W}_{i} / \bar{Z}}\right) . .
$$

As $\left.\varphi\right|_{Z}$ is an embedding, the Assumptions made in 3.1 hold and Theorem 3.3, i) and ii) and the projection formula imply that for all $i \in I$

$$
\operatorname{deg}_{\hat{\psi}_{i}^{*} \mathcal{L}}\left(\phi_{i}^{*} \check{N}_{\bar{W}_{i} / \bar{Z} / \text { torsion }}\right)<0 \text {. }
$$


Claim 4.7. If $\operatorname{dim}(Z)=d \geq 4$ then

$$
\operatorname{deg}_{\hat{\psi}_{i}^{*} \mathcal{L}}\left(\phi_{i}^{*} \check{N}_{\bar{W}_{i} / \bar{Z} / \text { torsion }}\right)=\left(\hat{\psi}_{i}\left(\hat{W}_{i}\right)\right)^{2} \cdot c_{1}(\mathcal{L})^{d-2} .
$$

In particular $\left(\hat{\psi}_{i}\left(\hat{W}_{i}\right)\right)^{2} \cdot c_{1}(\mathcal{L})^{d-2}<0$.

Proof. By (4.1) for any component $\Delta_{j}$ of $S_{\bar{W}_{i}}$ one has $\Delta_{j} \cdot \mathrm{c}_{1}(\mathcal{L})^{d-2}=0$, and the Claim 4.7 follows from (0.9).

Unfortunately, for $d \leq 3$ the corresponding equality is only guaranteed under the additional assumption made in the Notations 4.3, (2).

Claim 4.8. Assume that $\# I \geq \rho^{2}+\rho+1$. Then one of the following conditions hold:

a. There exists a linear combination

$$
D=\sum_{i \in I} a_{i} \cdot \hat{\psi}_{i}\left(\hat{W}_{i}\right)
$$

with $a_{i} \geq 0$, such that $D^{2} \cdot \mathrm{c}_{1}(\mathcal{L})^{d-2}>0$.

b. For all $i \in I$ the intersection number $\left(\hat{\psi}_{i}\left(\hat{W}_{i}\right)\right)^{2} \cdot \mathrm{c}_{1}(\mathcal{L})^{d-2} \leq 0$, and for some $\iota \in I$ and all $i \in I$ the intersection numbers

$$
\left(\hat{\psi}_{\iota}\left(\hat{W}_{\iota}\right)\right) \cdot\left(\hat{\psi}_{i}\left(\hat{W}_{i}\right)\right) \cdot \mathrm{c}_{1}(\mathcal{L})^{d-2}=0 .
$$

Proof. If $\left(\hat{\psi}_{i}\left(\hat{W}_{i}\right)\right)^{2} \cdot \mathrm{c}_{1}(\mathcal{L})^{d-2}>0$ for some $i \in I$ the condition a) obviously holds true. If there are two indices $i$ and $j$ with

$$
\left(\hat{\psi}_{\iota}\left(\hat{W}_{\iota}\right)\right) \cdot\left(\hat{\psi}_{i}\left(\hat{W}_{i}\right)\right) \cdot c_{1}(\mathcal{L})^{d-2}>0 \quad \text { and } \quad\left(\hat{\psi}_{\iota}\left(\hat{W}_{\iota}\right)\right)^{2} \cdot c_{1}(\mathcal{L})^{d-2}=0
$$

one can choose $D=\alpha \cdot \hat{\psi}_{\iota}\left(\hat{W}_{\iota}\right)+\hat{\psi}_{i}\left(\hat{W}_{i}\right)$ for $\alpha \gg 1$, and again a) holds.

If $\left(\hat{\psi}_{i}\left(\hat{W}_{i}\right)\right)^{2} \cdot c_{1}(\mathcal{L})^{d-2}<0$ for all $i \in I$, one can use Lemma 4.1 to verify a).

All the remaining cases are covered by b).

Claim 4.9. Assume that we are in case b) in Claim 4.8. Then there exists a blowing up $\Phi: \bar{Z}^{\prime} \rightarrow \bar{Z}$, which satisfies again the assumptions made in 4.2 , and for which one is in case a).

Proof. We choose $\Phi: \bar{Z}^{\prime} \rightarrow \bar{Z}$ to be the successive blowing up of the non-empty intersections $S_{\ell} \cap S_{k}$ of different components $S_{\ell}$ and $S_{k}$ of $S_{\bar{Z}}$. 
By Claim 4.7 the condition (1) in the Notations 4.3 excludes the case b) in Claim 4.8. For the other two conditions (2), and (3) stated there, the same is obvious. So the definition of $\varsigma(\bar{Z})$ and the assumptions made in Theorem 4.4, i) and ii) say that

$$
\# I \geq(\rho+\delta)^{2}+\rho+\delta+1=\rho\left(\bar{Z}^{\prime}\right)^{2}+\rho(\bar{Z})^{\prime}+1 .
$$

Of course we may assume that $\phi_{i}: \hat{W}_{i} \rightarrow \bar{W}_{i}$ is chosen such that $\hat{\psi}_{i}: \hat{W}_{i} \rightarrow \bar{Z}$ factors through $\hat{\psi}_{i}^{\prime}: \hat{W}_{i} \rightarrow \bar{Z}^{\prime}$.

As we had seen already in the proof of Proposition 4.5 the divisor

$$
S_{\bar{Z}^{\prime}}=\bar{Z}^{\prime} \backslash Z=\Phi^{-1}\left(S_{\bar{Z}}\right)
$$

is still a normal crossing divisor and

$$
\Omega_{\bar{Z}^{\prime}}^{1}\left(\log S_{\bar{Z}^{\prime}}\right)=\Phi^{*} \Omega_{\bar{Z}}^{1}\left(\log S_{\bar{Z}}\right) .
$$

So $\bar{Z}$ again satisfies the assumptions made in 4.2 .

Let $X \subset \bar{Z}$ be the smooth surface obtained by intersecting $d-2$ zero-divisors of general sections of $\mathcal{L}$ and let $X^{\prime}$ be its preimage in $\bar{Z}^{\prime}$. Then for all $i \in I$

$$
\left(\hat{\psi}_{i}\left(\hat{W}_{i}\right)\right)^{2} \cdot \mathrm{c}_{1}(\mathcal{L})^{d-2}=\left(\left.\hat{\psi}_{i}\left(\hat{W}_{i}\right)\right|_{X}\right)^{2} \geq\left(\left.\hat{\psi}_{i}^{\prime}\left(\hat{W}_{i}\right)\right|_{X^{\prime}}\right)^{2} .
$$

If this is an equality, none of the points lying on $\hat{\psi}_{i}\left(\hat{W}_{i}\right)$ is blown up.

On the other hand, if $\bar{W}_{i}$ is one of the divisors with $\left(\hat{\psi}_{i}\left(\hat{W}_{i}\right)\right)^{2} \cdot \mathrm{c}_{1}(\mathcal{L})^{d-2}=0$, hence with $\left(\left.\hat{\psi}_{i}\left(\hat{W}_{i}\right)\right|_{X}\right)^{2}=0$, then for some component $\Delta$ of $\hat{\psi}_{i}^{*} S_{\bar{Z}}-S_{\hat{W}_{i}}$ one has $\hat{\psi}_{i}(\Delta) \cdot \mathrm{c}_{1}(\mathcal{L})^{d-2}>0$. By Corollary $4.6 \hat{\psi}_{i}(\Delta)$ contains at least one of the intersections $S_{\ell, k}$, hence its restriction to $X$ is blown up.

Then for all $i \in I$ one finds $\left(\hat{\psi}_{i}^{\prime}\left(\hat{W}_{i}\right)\right)^{2} \cdot c_{1}\left(\Phi^{*} \mathcal{L}\right)^{d-2}<0$ and the last condition in Claim 4.8, b) is violated for $i=\iota$.

From now on, we will replace $\bar{Z}^{\prime}$ by $\bar{Z}$ and assume by abuse of notations that we are in case a) in Claim 4.8, hence for some effective linear combination $D$ of the $\psi_{i}\left(\bar{W}_{i}\right)$ we have $D^{2} . c_{1}(\mathcal{L})^{d-2}>0$.

Consider the saturated Higgs subsheaf $(F, \theta) \subset\left(E_{\bar{Z}}, \theta_{\bar{Z}}\right)$, which is generated by $E_{\bar{Z}}^{2,0}$, as described in (3.3) and (3.4). Since $(F, \theta) \subset\left(E_{\bar{Z}}, \theta_{\bar{Z}}\right)$ is a Higgs subbundle of a Higgs bundle arising from a variation of Hodge structures with logarithmic singularity along $S_{\bar{Z}}$, the sheaf $\operatorname{det}(F)$ is negative semi-definite in the sense that the curvature of the Hodge metric is negative semi-definite. 
Then by the projection formula

$$
\operatorname{deg}_{\hat{\psi}_{i}^{*} \mathcal{L}}\left(\phi_{i}^{*} \psi_{i}^{*} F / \text { torsion }\right)=\operatorname{deg}_{\psi_{i}^{*} \mathcal{L}}\left(\phi_{i_{*}} \phi_{i}^{*}\left(\psi_{i}^{*} F / \text { torsion }\right)\right) .
$$

Since $\psi_{i}^{*} F \hookrightarrow \phi_{i_{*}} \phi_{i}^{*}\left(\psi_{i}^{*} F /\right.$ torsion $) \hookrightarrow\left(\psi_{i}^{*} F\right)^{\natural}$, it follows from Addendum 3.4 that this degree is zero. The projection formula implies that

$$
\mathrm{c}_{1}(F) \cdot \hat{\psi}_{i}\left(\hat{W}_{i}\right) \cdot \mathrm{c}_{1}(\mathcal{L})^{d-2}=0 \quad \text { hence } \quad \mathrm{c}_{1}(F) \cdot D \cdot \mathrm{c}_{1}(\mathcal{L})^{d-2}=0 .
$$

Let again $X \subset \bar{Z}$ be the smooth surface obtained by intersecting $d-2$ zero-divisors of general sections of $\mathcal{L}$. Then $c_{1}\left(\left.F\right|_{X}\right) . D \cap X=0$. Since

$$
(D \cap X)^{2}>0 \quad \text { and } \quad\left(\left.\operatorname{det} F\right|_{X}\right)^{2} \geq 0,
$$

the Hodge index theorem implies that $\mathrm{c}_{1}\left(\left.\operatorname{det} F\right|_{X}\right)=0$.

Since $\mathrm{c}_{1}(F)$ is represented by a negative semi-definite Chern form the latter implies that $\mathrm{c}_{1}(F)=0$. By Simpson's poly-stability the Higgs subbundle $(F, \theta) \subset$ $(E, \theta)$ is a direct factor. Its complement has a trivial Higgs bundle, hence it is induced by a unitary local subsystem. By Lemma 1.5 we obtain i) and ii).

In Case iii) $\psi_{i}: \bar{W}_{i} \rightarrow \bar{Z}$ are morphisms. By assumption

$$
\left(\psi_{i} \bar{W}_{i}\right)^{2}=\operatorname{deg}\left(\check{N}_{\bar{W}_{i} / \bar{Z}}\right)=0 \quad \text { and } \quad \psi_{1}\left(\bar{W}_{1}\right) \cdot \psi_{2}\left(\bar{W}_{2}\right)>0 .
$$

Part iii) of the Addendum 3.4 implies that

$$
\mathrm{c}_{1}(F) \cdot\left(\psi_{1}\left(\bar{W}_{1}\right)+\psi_{2}\left(\bar{W}_{2}\right)\right)=\mathrm{c}_{1}\left(\left.F\right|_{\psi_{1}\left(\bar{W}_{1}\right)}\right)+\mathrm{c}_{1}\left(\left.F\right|_{\psi_{2}\left(\bar{W}_{2}\right)}\right)=0 .
$$

Since $c_{1}(F)$ is negative semi-definite and since $\left(\psi_{1}\left(\bar{W}_{1}\right)+\psi_{2}\left(\bar{W}_{2}\right)\right)^{2}>0$ the Hodge index theorem tells us that $\mathrm{c}_{1}(F)=0$. As before this implies that $(F, \theta)$ is a direct factor of $\left(E_{\bar{Z}}, \theta_{\bar{Z}}\right)$ and $Z \subset \mathcal{M}$ is a Shimura surface of Hodge type.

By Theorem 3.3, iii), the Griffiths-Yukawa coupling on $\bar{Z}$ does not vanish. Thus, $Z$ is a generalized Hilbert modular surface, necessarily rigid. $Z$ can not be a genuine Hilbert modular surface, since $Z$ contains Shimura curves with vanishing Griffiths-Yukawa coupling.

Proof of Theorems 0.2 and 0.3. As we have seen in the proof of Theorem 0.1 at the end of Section 2, on a surface $\bar{Y}=\bar{Z}$ the equality of Chern numbers (0.8) implies that the inequalities i), ii) and iii) in Theorem 3.3 coincide with the inequalities $(0.4),(0.5)$ and $(0.6)$ in Theorem 0.2. For the same reason, Theorem 0.3 is just a special case of Theorem 4.4 . 


\section{REFERENCES}

[Abd94] S. Abdulali: Conjugates of strongly equivariant maps, Pacific J. Math. 165 (1994), 207-216.

[An01] Y. AndrÉ: Shimura varieties, subvarieties, and CM points, I, Lecture, August 29, 2001.

[And07] F. ANDREATTA: Non-compact locally symmetric families of Jacobians, preprint 2007

[AMrT75] A. Ash, D. Mumford, M. RApoport, Y. TAI: Smooth compactification of locally symmetric varieties. Lie Groups: History, Frontiers and Applications, Vol. IV (1975).

[BHH87] G. BARThel, F. Hirzebruch AND T. Höfer: Geradenkonfigurationen und Algebraische Flächen, Aspects of Math. D4, Friedr. Vieweg \& Sohn, Braunschweig (1987).

[He62] S. Helgason: Differential Geometry and Symmetric Spaces, Academic Press, New York and London (1962).

[Hi58] F. Hirzebruch: Automorphe Formen und der Satz von Riemann-Roch, Symposium International der Topologia Algebraica, Unesco (1958).

[HZ73] F. HiRZEBruch: Hilbert modular surfaces, L'Ens. Math. 19 (1973), 57-113.

[KY06] B. Klingler And A. Yafaev: The André-Oort Conjecture, Preprint (2006).

[K05] S. Kondo: The moduli space of 5 points on $\mathbb{P}^{1}$ and $K 3$ surfaces, in "Arithmetic and geometry around hypergeometric functions", 189-206, Progr. Math. 260, Birkhäuser, Basel (2007).

[Kud03] S. Kudla: Special cycles and derivatives of Eisenstein series, Heegner points and Rankin L-series, 243-270, Math. Sci. Res. Inst. Publ., 49, Cambridge Univ. Press, Cambridge, (2004).

[KS67] M. Kuga, I. SATAKE: Abelian varieties attached to polarized K3 surfaces, Math. Annalen 169, 239-242 (1967).

[Lo03] E. LoOIJENGA: Compactifications defined by arrangements II: locally symmetric varieties of type IV, Duke Math. J. 119 (2003), 527-588.

[Mil04] J.S. MiLne: Introduction to Shimura Varieties, Preprint (2004), see www.jmilne.org/math/Preprints/svi.pdf.

[MVZ07] M. MöLler, E. VIeHweg AND K. Zuo: Stability of Hodge bundles and a numerical characterization of Shimura varieties, Preprint (2007).

[Mo98] Moonen, B.: Linearity properties of Shimura varieties, Part I. J. Algebraic Geom. 7 (1998), 539-567

[Mu77] D. MumFord: Hirzebruch's proportionality theorem in the non-compact case, Invent. math. 42 (1977), 239-272.

[SZ91] M. SAITo, S. ZuCKER: Classification of nonrigid families of K3 surfaces and a finiteness theorem of Arakelov type, Math. Ann. 289 (1991), 1-31.

[Si92] C. Simpson: Higgs bundles and local systems. Publ. Math. I.H.E.S. 75 (1992), 5-95.

[STZ03] X-T. Sun, S-L. TAN AND K. ZuO: Families of K3 surfaces satisfying Arakelov type equality, modular and Shimura curves. Mathematical Research Letters, 10 (2003), 323-342. 
[vG00] B. van GEEMEN: Kuga-Satake varieties and the Hodge conjecture, in "The arithmetic and geometry of algebraic cycles" (Banff, AB, 1998), 51-82, NATO Sci. Ser. C Math. Phys. Sci. 548, Kluwer Acad. Publ., Dordrecht (2000).

[VZ03] E. VIEHweg, K. ZuO: Families over curves with a strictly maximal Higgs field, Asian J. of Math. 7 (2003), 575-598.

[VZ04] E. Vienweg, K. Zuo: A characterization of certain Shimura curves in the moduli stack of abelian varieties, J. Differential Geometry 66 (2004), 233-287.

[VZ05] E. VIeHweg, K. ZuO: Arakelov inequalities and the uniformization of certain rigid Shimura varieties, J. Diff. Geom. 77 (2007) 291-352 preprint (2005), 56 pages, math.AG/0503339.

[Ya93] S.T. YAU: A splitting theorem and an algebraic geometric characterization of locally Hermitian symmetric spaces, Comm. in Analysis and Geom. 1 (1993), 473-486.

Stefan Müller-Stach

Universität Mainz, Fachbereich 08, Mathematik, 55099 Mainz, Germany

E-mail: mueller-stach@uni-mainz.de

Eckart Viehweg

Universität Duisburg-Essen, Mathematik, 45117 Essen, Germany

E-mail: viehweg@uni-due.de

Kang Zuo

Universität Mainz, Fachbereich 08, Mathematik, 55099 Mainz, Germany E-mail: zuok@uni-mainz.de 